\title{
Middle Convolution and Harnad Duality
}

\author{
Daisuke Yamakawa ${ }^{* \dagger}$ \\ CMLS, École polytechnique - CNRS UMR 7640 - \\ ANR SÉDIGA
}

\begin{abstract}
We interpret the additive middle convolution operation in terms of the Harnad duality, and as an application, generalize the operation to have a multiparameter and act on irregular singular systems.
\end{abstract}

\section{Introduction}

The middle convolution introduced by Katz [26] and reformulated by Völklein [40], Dettweiler-Reiter [17] is an operation acting on

- the category of local systems on a punctured projective line (in the multiplicative case); or

- that of Fuchsian systems (in the additive case).

The two multiplicative and additive operations match up via the Riemann-Hilbert correspondence [18]. Katz effectively used the middle convolution to study irreducible local systems which are rigid, namely, have no deformation preserving the local monodromy data, and proved that any such a local system is obtained by applying a finite iteration of tensor multiplications by rank 1 local systems and middle convolutions, to some rank 1 local system [26]. One can find many other applications of the middle convolution; in particular, to the Deligne-Simpson problem [15, 16, 28], to the classification/connection problems [38], and to the theory of isomonodromic deformations $[11,19,20,22]$.

${ }^{*}$ This research was supported by the grant ANR-08-BLAN-0317-01 of the Agence nationale de la recherche.

†current address: Département de mathématiques et applications, École normale supérieure, 45 rue d'Ulm, 75005 Paris, France; yamakawa@dma . ens . fr 
In this article we focus attention on the additive middle convolution. First recall its definition following Dettweiler-Reiter [17, Appendix]. Fix a finite set $D$ of points in $\mathbb{C}$ and suppose that a pair $(V, A)$ of a finite-dimensional $\mathbb{C}$-vector space $V$ and a Fuchsian system

$$
\frac{d u}{d z}=A(z) u, \quad A(z)=\sum_{t \in D} \frac{A_{t}}{z-t}, \quad A_{t} \in \operatorname{End}(V)
$$

with singularities on $D \cup\{\infty\}$ is given. Here we do not distinguish a system (1.1) and its coefficient matrix $A(z)$ fixing the coordinate $z$. The definition of the middle convolution $m c_{\lambda}(V, A)$ is divided into the following two steps.

(MC 1) Set $W_{t}:=V / \operatorname{Ker} A_{t}, t \in D$, and let

- $Q_{t}: W_{t} \rightarrow V$ be the injection induced from $A_{t}$; and

- $P_{t}: V \rightarrow W_{t}$ be the projection.

Obviously we have $A_{t}=Q_{t} P_{t}$. Set $W:=\bigoplus_{t \in D} W_{t}$ and let $Q: W \rightarrow V$ (resp. $P: V \rightarrow$ $W$ ) be the linear map whose block components with respect to the decomposition $W=\bigoplus W_{t}$ are $Q_{t}\left(\operatorname{resp} . P_{t}\right)$.

(MC 2) For $\lambda \in \mathbb{C}$, set $V^{\lambda}:=W / \operatorname{Ker}\left(P Q+\lambda \operatorname{Id}_{W}\right)$, and let

- $Q^{\lambda}: W \rightarrow V^{\lambda}$ be the projection; and

- $P^{\lambda}: V^{\lambda} \rightarrow W$ be the injection induced from $P Q+\lambda \operatorname{Id}_{W}$.

Obviously we have $P^{\lambda} Q^{\lambda}=P Q+\lambda \operatorname{Id}_{W}$. Let $Q_{t}^{\lambda}: W_{t} \rightarrow V^{\lambda}\left(\operatorname{resp} . P_{t}^{\lambda}: V^{\lambda} \rightarrow W_{t}\right)$ be the block components of $Q^{\lambda}$ (resp. $\left.P^{\lambda}\right)$.

Definition 1.1 (Dettweiler-Reiter). We call

$$
m c_{\lambda}(V, A):=\left(V^{\lambda}, A^{\lambda}\right), \quad A^{\lambda}(z)=\sum_{t \in D} \frac{Q_{t}^{\lambda} P_{t}^{\lambda}}{z-t}
$$

the (additive) middle convolution of $(V, A)$ with $\lambda$.

Here looking at the above procedure, one can observe that the given pair $(V, A)$ and its middle convolution are described as

$$
A(z)=Q\left(z \operatorname{Id}_{W}-T\right)^{-1} P, \quad A^{\lambda}(z)=Q^{\lambda}\left(z \operatorname{Id}_{W}-T\right)^{-1} P^{\lambda},
$$

where $T:=\bigoplus_{t} t \operatorname{Id}_{W_{t}} \in \operatorname{End}(W)$. Such an expression of a system can be found in the papers of Adams, Harnad, Hurtubise and Previato [1-3, 21] and implicitly in that 
of Jimbo-Miwa-Môri-Sato [25]. In particular, Harnad [21] considered two systems having the following symmetric description:

$$
A(z)=S+Q\left(z \operatorname{Id}_{W}-T\right)^{-1} P, \quad B(\zeta)=T+P\left(\zeta \operatorname{Id}_{V}-S\right)^{-1} Q,
$$

where $Q \in \operatorname{Hom}(W, V), P \in \operatorname{Hom}(V, W)$, and $S, T$ are semisimple endomorphisms of $V, W$ respectively. He then obtained an equivalence (called the Harnad duality) between the isomonodromic deformations of the systems $A(z)$ and $-B(\zeta)$. Note that if $S=0$, we have $B(\zeta)=T+P Q \zeta^{-1}$. So the procedure getting the middle convolution can be rephrased roughly as follows: for given Fuchsian system $A(z)=Q\left(z \operatorname{Id}_{W}-\right.$ $T)^{-1} P$,

(a) take its 'Harnad dual' $11 B(\zeta)=T+P Q \zeta^{-1}$;

(b) shift $B(\zeta)$ by $\lambda \zeta^{-1}$; and then

(c) take the Harnad dual again.

Such a relation between the middle convolution and the Harnad duality is already known by Boalch [11, 12]. It may be viewed as another formulation of Katz's interpretation of the middle convolution via Fourier transform $[26, \S 2.10]$. Note that the above procedure makes sense even in the case that $S$ is an arbitrary semisimple endomorphism. Suppose that a system $A(z)=S+\sum A_{t} /(z-t)$ with simple poles on $D$ and a pole of order 2 at $\infty$ is given. Then at step (a), take its Harnad dual $B(\zeta)=T+P\left(\zeta \operatorname{Id}_{V}-S\right)^{-1} Q$. Next at step (b), shift it by some rank 1 Fuchsian system $\alpha(\zeta)$ having singularities at the eigenvalues of $S$. Finally at step (c), take the Harnad dual again. Then we get the middle convolution $m c_{\alpha}(A)$ with $\alpha$. Boalch generalized the Harnad duality, called the cycling, and obtained a further generalization of the middle convolution (see [13, §4.6]) for systems with simple poles at $D$ and a pole of order 3 at $\infty$ which has a 'normal form' (see Definition 6.7).

If $T$ is not semisimple, then the matrix-valued function $Q\left(z \operatorname{Id}_{W}-T\right)^{-1} P$ has in general higher order poles at the eigenvalues of $T$. In fact, it is known [27, 42] that for any system of the form

$$
A(z)=\sum_{t \in D} \sum_{k=1}^{k_{t}} \frac{A_{t, k}}{(z-t)^{k}}, \quad A_{t, k} \in \operatorname{End}(V), \quad k_{t} \in \mathbb{Z}_{>0},
$$

there exist a finite-dimensional $\mathbb{C}$-vector space $W$, an endomorphism $T$ of $W$ and homomorphisms $Q: W \rightarrow V$ and $P: V \rightarrow W$, such that $A(z)=Q\left(z \operatorname{Id}_{W}-T\right)^{-1} P$. One

\footnotetext{
${ }^{1}$ For convenience, we use the terminology 'Harnad dual' on the system $B(\zeta)$, not on $-B(\zeta)$, while the original 'Harnad duality' is the correspondence between $A(z)$ and $-B(\zeta)$.
} 
may then expect that the middle convolution operation $m c_{\alpha}$ can be generalized to that acting on systems of the form (1.2). In order to obtain such a generalization, we have to make a rigorous meaning of the 'Harnad dual', because for given system $A(z)$, the choice of datum $(W, T, Q, P)$ satisfying $A(z)=Q\left(z \mathrm{Id}_{W}-T\right)^{-1} P$ is not unique, so we have to eliminate ambiguity of the choice in a certain canonical way. In Fuchsian case, what we do in (MC 1) gives the answer to it, so the problem is easily solved. In this article, as a generalization of the procedure (MC 1), we give an explicit construction of $(W, T, Q, P)$ for any system $A(z)$ of the form (1.2), and show that it is 'canonical' in the following sense: the constructed datum $(W, T, Q, P)$ together with $V$, which we call the canonical datum for $A(z)$ (Definition 4.6), satisfies a stability condition (Definition 3.3) in the sense of Mumford's geometric invariant theory, and is characterized up to isomorphism via this condition. More precisely, we show the following:

Theorem 1.2 (Proposition 4.1 and Proposition 4.7). For any system A(z) of the form as in (1.2) with $V \neq 0$, the canonical datum is stable; in particular, there exists a stable datum $(V, W, T, Q, P)$ satisfying $Q\left(z \operatorname{Id}_{W}-T\right)^{-1} P=A(z)$.

If two data $(V, W, T, Q, P)$ and $\left(V, W^{\prime}, T^{\prime}, Q^{\prime}, P^{\prime}\right)$ with the same $V \neq 0$ are both stable and satisfy

$$
Q\left(z \operatorname{Id}_{W}-T\right)^{-1} P=Q^{\prime}\left(z \operatorname{Id}_{W^{\prime}}-T^{\prime}\right)^{-1} P^{\prime},
$$

then there exists an isomorphism $f: W \rightarrow W^{\prime}$ such that

$$
Q^{\prime}=Q f^{-1}, \quad P^{\prime}=f P, \quad T^{\prime}=f T f^{-1} .
$$

Using the canonical data, we can define the notion of Harnad dual as follows: for given system of the form

$$
A(z)=S+A^{0}(z), \quad A^{0}(z)=\sum_{t \in D} \sum_{k=1}^{k_{t}} \frac{A_{t, k}}{(z-t)^{k}}, \quad S, A_{t, k} \in \operatorname{End}(V), \quad k_{t} \in \mathbb{Z}_{>0},
$$

take the canonical datum $(V, W, T, Q, P)$ for the system $A^{0}(z)$, and set $B(\zeta):=T+$ $P\left(\zeta \operatorname{Id}_{V}-S\right)^{-1} Q$. We call the pair $(W, B)$ as the Harnad dual of $(V, A)$ and denote it by $\operatorname{HD}(V, A)$ (Definition 5.2). Then the above characterization in terms of the geometric invariant theory gives the following two basic properties of HD:

Theorem 1.3 (Theorem 5.8). Suppose that a pair $(V, A)$ of the form as in (1.3) with $V \neq 0$ satisfies the following two conditions:

(a) $(V, A)$ is irreducible, namely, $V$ has no nonzero proper subspace preserved by all $A_{t, k}$ and $S$; 
(b) $(V, A)$ is not equivalent to a pair of the form $(\mathbb{C}, s), s \in \mathbb{C}$, under constant gauge transformation.

Then $\operatorname{HD}(V, A)$ is also irreducible and $\operatorname{HD} \circ \operatorname{HD}(V, A)$ is equivalent to $(V, A)$ under constant gauge transformation.

Theorem 1.4 (see Theorem 5.10 for the rigorous statement). The correspondence between $(V, A)$ and $(W,-B)$, where $(W, B)=\mathrm{HD}(V, A)$, gives a symplectomorphism between naive moduli spaces of irreducible systems of the form as in (1.3) having different singularities with different truncated formal types.

Now the middle convolution (Definition 6.1) is defined as

$$
m c_{\alpha}(V, A):=\mathrm{HD} \circ a d d_{\alpha} \circ \mathrm{HD}(V, A)
$$

where the parameter $\alpha(\zeta)$ is a rank 1 system having singularities at the eigenvalues of $S=\lim _{z \rightarrow \infty} A(z)$, and $a d d_{\alpha}:(W, B) \mapsto(W, B+\alpha)$ is the addition operator. Note that here we do not require that $S, T$ are semisimple or $\alpha$ is Fuchsian. We obtain the following properties of $m c_{\alpha}$, which are well-known in the original case, as corollaries of the above two results on HD:

Corollary 1.5 (Corollary6.3 and Corollary 6.4). Suppose that a pair $(V, A)$ of the form as in (1.3) satisfies Conditions (a) and (b) in Theorem 1.3. Then $m c_{0}(V, A) \sim(V, A)$. Furthermore, if a rank 1 system $\alpha(\zeta)$ with singularities at the eigenvalues of $S$ satisfies $m c_{\alpha}(V, A) \neq(0,0)$, then $m c_{\alpha}(V, A)$ is also irreducible and

$$
m c_{\beta} \circ m c_{\alpha}(V, A) \sim m c_{\alpha+\beta}(V, A)
$$

for any $\beta$.

Corollary 1.6 (see Corollary 6.5 for the rigorous statement). The middle convolution $m c_{\alpha}$ gives a symplectomorphism between naive moduli spaces of irreducible systems of the form as in (1.3) having different singularities with different truncated formal types.

Arinkin also generalized Katz's middle convolution to the irregular singular case in $\mathcal{D}$-module setting [4], and generalized Katz's algorithm by adding the Fourier transform to the original one [5]. We show using our generalized middle convolution that Katz's algorithm works well for 'naively rigid systems having a normal form' (see Definition 7.1 and Definition 6.7), which corresponds to the case that the Fourier transform is not necessary in Arinkin's generalized algorithm: 
Theorem 1.7 (Corollary 7.6). Suppose that a pair $(V, A)$ of the form as in (1.2) with $\operatorname{dim} V \geq 2, \operatorname{Res}_{z=\infty} A(z)=0$ is irreducible, naively rigid, and has a normal form at any $t \in D$. Then applying a suitable finite iteration of operations of the form

$$
a d d_{\alpha} \circ m c_{\lambda / \zeta} \circ a d d_{\alpha}, \quad \alpha \in \mathcal{E}_{\vec{k}}(\mathbb{C}), \lambda=\operatorname{Res}_{z=\infty} \alpha(z),
$$

makes $(V, A)$ into an irreducible pair of rank 1.

\section{Setting}

For a positive integer $k$, we set

$$
S_{k}:=\mathbb{C}[z] / z^{k} \mathbb{C}[z], \quad S^{k}:=z^{-k} \mathbb{C}[z] / \mathbb{C}[z] .
$$

$S_{k}$ is a $k$-dimensional $\mathbb{C}$-algebra, and the pairing

$$
S_{k} \otimes_{\mathbb{C}} S^{k} \rightarrow \mathbb{C} ; \quad(f, g) \mapsto \operatorname{Res}_{z=0}(f(z) g(z))
$$

gives an identification $S_{k}^{*}=\operatorname{Hom}_{\mathbb{C}}\left(S_{k}, \mathbb{C}\right) \simeq S^{k}$.

For a finite-dimensional $\mathbb{C}$-vector space $V$, set

$$
G_{k}(V):=\operatorname{Aut}_{S_{k}}\left(S_{k} \otimes_{\mathbb{C}} V\right), \quad \mathfrak{g}_{k}(V):=\operatorname{End}_{S_{k}}\left(S_{k} \otimes_{\mathbb{C}} V\right)
$$

Any element of $\mathfrak{g}_{k}(V)$ is uniquely written as

$$
\xi_{0}+\xi_{1} z+\cdots+\xi_{k-1} z^{k-1}, \quad \xi_{i} \in \operatorname{End}(V)
$$

and $G_{k}(V)$ is just the subset of $\mathfrak{g}_{k}(V)$ defined by the condition $\operatorname{det} \xi_{0} \neq 0$. It has naturally a structure of complex algebraic group and the associated Lie algebra is nothing but $\mathfrak{g}_{k}(V)$. The $\mathbb{C}$-dual $\mathfrak{g}_{k}^{*}(V)$ of $\mathfrak{g}_{k}(V)$ can be identified with the set

$$
\left\{\eta_{1} z^{-1}+\eta_{2} z^{-2}+\cdots+\eta_{k} z^{-k} \mid \eta_{i} \in \operatorname{End}(V)\right\} .
$$

For $\eta=\sum_{j} \eta_{j} z^{-j} \in \mathfrak{g}_{k}^{*}(V)$, we denote by $\operatorname{ord}(\eta)$ the pole order of $\eta$;

$$
\operatorname{ord}(\eta):=\max \left(\left\{j \geq 1 \mid \eta_{j} \neq 0\right\} \cup\{0\}\right),
$$

which is preserved under the $G_{k}(V)$-coadjoint action.

For a collection $\vec{k}=\left(k_{t}\right)_{t \in D}$ of positive integers indexed by a finite set $D$ of points in $\mathbb{C}$, we set

$$
\mathcal{E}_{\vec{k}}(V):=\left\{A(z)=\sum_{t \in D} \sum_{k=1}^{k_{t}} \frac{A_{t, k}}{(z-t)^{k}} \mid A_{t, k} \in \operatorname{End}(V)\right\},
$$


and regard $A(z) \in \mathcal{E}_{\vec{k}}(V)$ as a system of linear ordinary differential equations

$$
\frac{d u}{d z}=A(z) u
$$

with singularities on $D \cup\{\infty\}$. If we define

$$
G_{\vec{k}}(V):=\prod_{t \in D} G_{k_{t}}(V), \quad \mathfrak{g}_{\vec{k}}(V):=\bigoplus_{t \in D} \mathfrak{g}_{k_{t}}(V), \quad \mathfrak{g}_{\vec{k}}^{*}(V):=\bigoplus_{t \in D} \mathfrak{g}_{k_{t}}^{*}(V),
$$

then $\mathcal{E}_{\vec{k}}(V)$ is isomorphic to $\mathfrak{g}_{\vec{k}}^{*}(V)$ by the map

$$
\mathcal{E}_{\vec{k}}(V) \stackrel{\sim}{\rightarrow} \mathfrak{g}_{\vec{k}}^{*}(V) ; \quad A(z) \mapsto\left(\sum A_{t, k} z^{-k}\right)_{t \in D},
$$

and hence $G_{\vec{k}}(V)$ naturally acts on $\mathcal{E}_{\vec{k}}(V)$.

Now for two finite-dimensional $\mathbb{C}$-vector spaces $V$ and $W$, we set

$$
\mathbf{M}(V, W):=\operatorname{Hom}(W, V) \oplus \operatorname{Hom}(V, W) .
$$

It has a natural symplectic structure

$$
\omega=\operatorname{tr} d Q \wedge d P, \quad(Q, P) \in \mathbf{M}(V, W),
$$

and the group $\mathrm{GL}(V) \times \mathrm{GL}(W)$ acts symplectomorphically on $\mathbf{M}(V, W)$ by

$$
(a, b) \cdot(Q, P):=\left(a Q b^{-1}, b P a^{-1}\right), \quad(a, b) \in \mathrm{GL}(V) \times \mathrm{GL}(W) .
$$

Note that the map $\left(\mu_{V}, \mu_{W}\right): \mathbf{M}(V, W) \rightarrow \mathfrak{g l}(V) \oplus \mathfrak{g l}(W)$ defined by

$$
\mu_{V}(Q, P):=Q P, \quad \mu_{W}(Q, P):=-P Q,
$$

is a moment map generating the $\operatorname{GL}(V) \times \mathrm{GL}(W)$-action, where we identify the Lie algebras $\mathfrak{g l}(V), \mathfrak{g l}(W)$ with those duals via the trace pairing. Let $T \in \operatorname{End}(W)$ be an endomorphism with eigenvalues in $D$ and $W=\bigoplus_{t \in D} W_{t}$ be its generalized eigenspace decomposition. Let $N_{t}:=\left.T\right|_{W_{t}}-t \operatorname{Id}_{W_{t}} \in \operatorname{End}\left(W_{t}\right)$ be the nilpotent part of $T$ restricted to $W_{t}$. Then we can consider the map

$$
\Phi_{T}: \mathbf{M}(V, W) \rightarrow \mathcal{E}_{\vec{k}}(V) ; \quad(Q, P) \mapsto Q\left(z \operatorname{Id}_{W}-T\right)^{-1} P,
$$

where $k_{t}=\min \left\{j \in \mathbb{Z}_{>0} ; N_{t}^{j}=0\right\}$. To see it is well-defined, for $(Q, P) \in \mathbf{M}(V, W)$, let $\left(Q_{t}, P_{t}\right) \in \mathbf{M}\left(V, W_{t}\right)$ be its $\mathbf{M}\left(V, W_{t}\right)$-component with respect to the decomposition $\mathbf{M}(V, W)=\bigoplus \mathbf{M}\left(V, W_{t}\right)$ induced from $W=\bigoplus W_{t}$. Then we have

$$
\begin{aligned}
Q\left(z \operatorname{Id}_{W}-T\right)^{-1} P & =\sum_{t \in D} Q_{t}\left[(z-t) \operatorname{Id}_{W_{t}}-N_{t}\right]^{-1} P_{t} \\
& =\sum_{t \in D}(z-t)^{-1} Q_{t}\left[\operatorname{Id}_{W_{t}}-(z-t)^{-1} N_{t}\right]^{-1} P_{t} \\
& =\sum_{t \in D} \sum_{k=1}^{k_{t}} \frac{Q_{t} N_{t}^{k-1} P_{t}}{(z-t)^{k}} .
\end{aligned}
$$

Therefore $\Phi_{T}(Q, P)$ can be considered as an element of $\mathcal{E}_{\vec{k}}(V)$. 
Theorem 2.1 (Adams-Harnad-Hurtubise-Previato). For $g=\left(g_{t}(z)\right) \in G_{\vec{k}}(V)$ and $(Q, P) \in \mathbf{M}(V, W)$, let $(g \cdot Q, g \cdot P) \in \mathbf{M}(V, W)$ be the point given by the following formulae:

$$
\begin{aligned}
g_{t}(z) Q_{t}\left(z-N_{t}\right)^{-1} & =(g \cdot Q)_{t}\left(z-N_{t}\right)^{-1}+\text { holomorphic, } \\
\left(z-N_{t}\right)^{-1} P_{t} g_{t}^{-1}(z) & =\left(z-N_{t}\right)^{-1}(g \cdot P)_{t}+\text { holomorphic. }
\end{aligned}
$$

Then it gives a well-defined Hamiltonian action of $G_{\vec{k}}(V)$ on $\mathbf{M}(V, W)$ with moment $\operatorname{map} \Phi_{T}$.

More explicitly, $(g \cdot Q, g \cdot P)$ is defined by

$$
(g \cdot Q)_{t} \equiv g_{t} \cdot Q_{t}:=\sum_{k=0}^{k_{t}-1} g_{t, k} Q_{t} N_{t}^{k}, \quad(g \cdot P)_{t} \equiv g_{t} \cdot P_{t}:=\sum_{k=0}^{k_{t}-1} N_{t}^{k} P_{t}\left(g_{t}^{-1}\right)_{k},
$$

where $g_{t}(z)=\sum g_{t, k} z^{k}, g_{t}^{-1}(z)=\sum\left(g_{t}^{-1}\right)_{k} z^{k}$. Note that the above gives actions on $\operatorname{Hom}(W, V)$ and $\operatorname{Hom}(V, W)$ separately and the two are coadjoint to each other.

The proof was given by Adams-Harnad-Previato [3] in the case that $T$ is semisimple and by Adams-Harnad-Hurtubise [1] in general cases. Strictly speaking, the original result is stated in terms of loop group action, however one can easily derive the above from it.

Recall that any moment map is Poisson. Therefore the above theorem connects two Poisson manifolds $\mathbf{M}(V, W)$ and $\mathcal{E}_{\vec{k}}(V)$, the space of systems of linear differential equations, via $\Phi_{T}$.

Now, take one more finite set $E$ of points in $\mathbb{C}$ and a collection $\vec{l}=\left(l_{s}\right)_{s \in E}$ of positive integers indexed by $E$. Let $S$ be an endomorphism of $V$ with eigenvalues in $E$ and $V=\bigoplus_{s \in E} V_{s}$ be its generalized eigenspace decomposition. For any $s \in E$, set $M_{s}:=\left.S\right|_{V_{s}}-s \operatorname{Id}_{V_{s}}$ and suppose $M_{s}^{l_{s}}=0$. Then we can also define

$$
\Psi_{S}: \mathbf{M}(V, W) \rightarrow \mathcal{E}_{l}(W), \quad(Q, P) \mapsto-P\left(\zeta \mathrm{Id}_{V}-S\right)^{-1} Q,
$$

where we denote the indeterminate by $\zeta$ instead of $z$. It is a moment map generating the following $G_{\vec{l}}(W)$-action on $\mathbf{M}(V, W)$ :

$$
(h \cdot Q)_{s} \equiv h_{s} \cdot Q_{s}:=\sum_{l=0}^{l_{s}-1} M_{s}^{l} Q_{s}\left(h_{s}^{-1}\right)_{l}, \quad(h \cdot P)_{s} \equiv h_{s} \cdot P_{s}:=\sum_{l=0}^{l_{s}-1} h_{s, l} P_{s} M_{s}^{l},
$$

where $\left(Q_{s}, P_{s}\right)$ is the $\mathbf{M}\left(V_{s}, W\right)$-component of $(Q, P)$ and

$$
h=\left(h_{s}\right)_{s \in E} \in G_{l}(W), \quad h_{s}(\zeta)=\sum_{l=0}^{l_{s}} h_{s, l} \zeta^{l}, h_{s}^{-1}(\zeta)=\sum_{l=0}^{l_{s}}\left(h_{s}^{-1}\right)_{l} \zeta^{l} .
$$

Throughout this article, we fix two nonempty finite sets $D, E$ of points in $\mathbb{C}$, and collections $\vec{k}=\left(k_{t}\right)_{t \in D}, \vec{l}=\left(l_{s}\right)_{s \in E}$ of positive integers indexed by $D, E$ respectively. 


\section{Preliminary results from GIT}

Recall that a quiver is a quadruple $Q=(I, \Omega$, out, in) consisting of two sets $I, \Omega$ and two maps out, in: $\Omega \rightarrow I$. The sets $I, \Omega$ are called the set of vertices, the set of arrows respectively, and each $h \in \Omega$ is viewed as an arrow drawn from the vertex $\operatorname{out}(h)$ to the vertex $\operatorname{in}(h)$.

A representation of the quiver $Q$ is a pair consisting of a collection of $\mathbb{C}$-vector spaces $V_{i}$ indexed by $i \in I$, and a collection of linear maps $x_{h}: V_{\text {out }(h)} \rightarrow V_{\text {in }(h)}$ indexed by $h \in \Omega$. For two representations given by $V_{i}^{\prime}, x_{h}^{\prime}$ and $V_{i}, x_{h}$, a morphism from the former to the latter is a collection of linear maps $\psi_{i}: V_{i}^{\prime} \rightarrow V_{i}$ indexed by $i \in I$, such that $\psi_{\operatorname{in}(h)} \circ x_{h}^{\prime}=x_{h} \circ \psi_{\text {out }(h)}$ for all $h \in \Omega$. It is called an isomorphism if each $\psi_{i}$ is an isomorphism. If each $\psi_{i}$ is just an injection, the collection $\left(\operatorname{Im} \psi_{i}\right)_{i \in I}$ together with

the linear maps $\operatorname{Im} \psi_{\text {out }(h)} \stackrel{x_{h}}{\rightarrow} \operatorname{Im} \psi_{\text {in }(h)}$ induced from $x_{h}$ gives a representation of $\boldsymbol{Q}$, which is called a subrepresentation of $\left(V_{i}, x_{h}\right)$. Note that subspaces $X_{i} \subset V_{i}, i \in I$ give a subrepresentation of $\left(V_{i}, x_{h}\right)$ if and only if $x_{h}\left(X_{\text {out }(h)}\right) \subset X_{\text {in }(h)}$ for all $h \in \Omega$.

For a positive integer $r \in \mathbb{Z}_{>0}$, let $Q_{r}$ be the quiver with set of vertices $D \cup\{\infty\}$ obtained by drawing $r$ arrows both from $t$ to $\infty$ and $\infty$ to $t$, and an edge-loop (i.e., an arrow $h$ with $\operatorname{in}(h)=\operatorname{out}(h))$ at $t$ for each $t \in D$. Let $Q \equiv Q_{1}$ for simplicity.

Each quintuple $(V, W, T, Q, P)$ consisting of

- two finite-dimensional $\mathbb{C}$-vector spaces $V, W$;

- an endomorphism $T$ of $W$ whose eigenvalues are all contained in $D$; and

- a point $(Q, P) \in \mathbf{M}(V, W)$

gives a representation of $Q$ in the following way:

(a) for each $t \in D$, assign the vertex $t$ with the vector space $W_{t}:=\operatorname{Ker}(T-$ $\left.t \operatorname{Id}_{W}\right)^{\operatorname{dim} W}$, and assign the vertex $\infty$ with $V$;

(b) assign the arrow from $t$ to $\infty$ with $Q_{t}$, the $\operatorname{Hom}\left(W_{t}, V\right)$-block component of $Q$; and similarly,

(c) assign the arrow from $\infty$ to $t$ with $P_{t} \in \operatorname{Hom}\left(V, W_{t}\right)$;

(d) assign the loop at $t$ with $N_{t}:=\left.\left(T-t \mathrm{Id}_{W}\right)\right|_{W_{t}}$.

We call such a quintuple $(V, W, T, Q, P)$ satisfying further the following condition just as a datum:

$$
\prod_{t \in D}\left(T-t \operatorname{Id}_{W}\right)^{k_{t}}=0,
$$


where the zero datum $(0,0,0,0,0)$, which corresponds to the zero representation of $Q$, is understood to satisfy the above.

Definition 3.1. A subrepresentation of a datum $(V, W, T, Q, P)$ is a pair $(X, Y)$ of vector subspaces $X \subset V, Y \subset W$ satisfying that

$$
P(X) \subset Y, \quad Q(Y) \subset X, \quad T(Y) \subset Y .
$$

If $(X, Y)$ is a subrepresentation of a datum $(V, W, T, Q, P)$, then we can consider the restriction $\left(X, Y,\left.T\right|_{Y},\left.Q\right|_{Y},\left.P\right|_{X}\right)$ of the datum to the subspaces $X$ and $Y$, which obviously gives a subrepresentation of the representation of $Q$ corresponding to $(V, W, T, Q, P)$, and vice versa.

Representations of $Q$ with prescribed vector spaces $V$ at $\infty$ and $W_{t}$ at $t \in D$ form a vector space

$$
\operatorname{Rep}_{Q}(V, \mathbf{W}):=\bigoplus_{t \in D}\left(\mathbf{M}\left(V, W_{t}\right) \oplus \operatorname{End}\left(W_{t}\right)\right), \quad \mathbf{W}=\left(W_{t}\right)_{t \in D}
$$

and the group $\mathrm{GL}(V) \times \mathrm{GL}(\mathbf{W})$, where $\mathrm{GL}(\mathbf{W}):=\prod_{t \in D} \mathrm{GL}\left(W_{t}\right)$, acts on it as isomorphisms of representations.

Definition 3.2. A nonzero datum $(V, W, T, Q, P)$ is said to be irreducible if it has no subrepresentations except $(X, Y)=(0,0),(V, W)$, or equivalently, if the corresponding representation of $Q$ is irreducible.

We also need the following condition.

Definition 3.3. A datum $(V, W, T, Q, P)$ with $V \neq 0$ is said to be stable if for any subrepresentation $(X, Y)$ of it, the equalities $X=0, V$ imply $Y=0, W$ respectively.

Note that under the assumption $V \neq 0$, the irreducibility condition implies the stability condition.

Remark 3.4. Let us fix $V \neq 0$ and consider the quiver $Q_{\operatorname{dim} V}$. Representations of $Q_{\operatorname{dim} V}$ with prescribed vector spaces $\mathbb{C}$ at $\infty$ and $W_{t}$ at $t \in D$, form a vector space

$$
\operatorname{Rep}_{Q_{\operatorname{dim} V}}(\mathbb{C}, \mathbf{W})=\bigoplus_{t \in D}\left(\mathbf{M}\left(\mathbb{C}, W_{t}\right)^{\oplus \operatorname{dim} V} \oplus \operatorname{End}\left(W_{t}\right)\right)
$$

On the other hand, fixing a basis of $V$, we have identifications

$$
\operatorname{Hom}\left(W_{t}, V\right) \simeq \operatorname{Hom}\left(W_{t}, \mathbb{C}\right)^{\oplus \operatorname{dim} V}, \quad \operatorname{Hom}\left(V, W_{t}\right) \simeq \operatorname{Hom}\left(\mathbb{C}, W_{t}\right)^{\oplus \operatorname{dim} V}
$$


Thus we have an isomorphism

$$
\operatorname{Rep}_{Q}(V, \mathbf{W}) \simeq \operatorname{Rep}_{Q_{\operatorname{dim} V}}(\mathbb{C}, \mathbf{W})
$$

which enables us to regard any datum with fixed $V$ as a representation of the quiver $Q_{\operatorname{dim} V}$, of which the vector space at the vertex $\infty$ is just $\mathbb{C}$.

Now fix a datum $(V, W, T, Q, P)$ and suppose that a subrepresentation of the corresponding representation of $Q_{\operatorname{dim} V}$ is given. Then one of the following two cases occurs:

(a) the vector space of it at the vertex $\infty$ is 0 ;

(b) the vector space of it at the vertex $\infty$ is $\mathbb{C}$.

In the first case, the subrepresentation gives a vector subspace $Y_{t} \subset W_{t}$ for each $t \in D$ such that

$$
Q_{t}\left(Y_{t}\right)=0, \quad N_{t}\left(Y_{t}\right) \subset Y_{t},
$$

namely, the pair $(0, Y), Y=\bigoplus_{t \in D} Y_{t}$ is a subrepresentation of $(V, W, T, Q, P)$. In the second case, the subrepresentation gives a vector subspace $Y_{t} \subset W_{t}$ for each $t \in D$ such that

$$
P_{t}(V) \subset Y_{t}, \quad N_{t}\left(Y_{t}\right) \subset Y_{t},
$$

namely, the pair $(V, Y), Y=\bigoplus_{t \in D} Y_{t}$ is a subrepresentation of $(V, W, T, Q, P)$.

This observation shows that when $V \neq 0$, a datum $(V, W, T, Q, P)$ is stable if and only if the corresponding representation of $Q_{\operatorname{dim} V}$ is irreducible.

Lemma 3.5. Let $\left(Q_{t}, P_{t}, N_{t}\right)_{t \in D} \in \operatorname{Rep}_{Q}(V, \mathbf{W})$ be the point corresponding to some datum $(V, W, T, Q, P)$. If $V \neq 0$ and $(V, W, T, Q, P)$ is stable, then the stabilizer at the point $\left(Q_{t}, P_{t}, N_{t}\right)_{t \in D} \in \operatorname{Rep}_{Q}(V, \mathbf{W})$ of $\mathrm{GL}(\mathbf{W})$ is trivial.

Proof. Suppose that $b=\left(b_{t}\right) \in \mathrm{GL}(\mathbf{W})$ stabilizes $\left(Q_{t}, P_{t}, N_{t}\right)_{t \in D}$, namely,

$$
Q_{t} b_{t}^{-1}=Q_{t}, \quad b_{t} P_{t}=P_{t}, \quad b_{t} N_{t} b_{t}^{-1}=N_{t}
$$

Set $Y_{t}:=\operatorname{Ker}\left(b_{t}-1\right) \subset W_{t}$ and $Y:=\bigoplus Y_{t}$. Then the above implies that

$$
N_{t}\left(Y_{t}\right) \subset Y_{t}, \quad \operatorname{Im} P_{t} \subset Y_{t}
$$

Thus $(V, Y)$ is a subrepresentation of $(V, W, T, Q, P)$. By the stability condition we must have $Y=W$ and hence $b=1$. 
Lemma 3.6. Suppose that $V \neq 0$ and let $\left(Q_{t}, P_{t}, N_{t}\right)_{t \in D} \in \operatorname{Rep}_{Q}(V, \mathbf{W})$ be the point corresponding to some datum $(V, W, T, Q, P)$. Then the $\mathrm{GL}(\mathbf{W})$-orbit of it is closed if and only if there exists a direct sum decomposition

$$
W=W(0) \oplus W(1)^{\oplus m_{1}} \oplus \cdots \oplus W(N)^{\oplus m_{N}}
$$

compatible with the decomposition $W=\bigoplus_{t} W_{t}$ such that:

(a) $Q\left(W(i)^{\oplus m_{i}}\right)=0$ for all $i \geq 1$ and $\operatorname{Im} P \subset W(0)$;

(b) T preserves each direct summand;

(c) for any $i \geq 1$ there exists $T_{i} \in \operatorname{End}(W(i))$ such that $\left.T\right|_{W(i)^{\oplus m_{i}}}=\operatorname{Id}_{\mathbb{C}^{m_{i}}} \otimes T_{i}$;

(d) the datum $\left(V, W(0),\left.T\right|_{W(0)}, Q, P\right)$ given by restricting $(V, W, T, Q, P)$ to $W(0)$ is stable;

(e) the datum $\left(0, W(i), T_{i}, 0,0\right)$ is irreducible for any $i \geq 1$.

Moreover such a direct decomposition is unique up to permutation on $\{1, \ldots, N\}$.

Proof. Let $x \in \operatorname{Rep}_{Q_{\operatorname{dim} V}}(\mathbb{C}, \mathbf{W})$ be the point corresponding to $(V, W, T, Q, P)$ under the isomorphism given in Remark 3.4. It is well-known [30] that the orbit GL(W) $x$ is closed if and only if $x$ is semisimple as a representation of $Q_{\operatorname{dim} V}$, namely there exists a direct sum decomposition

$$
x=x(0)^{\oplus m_{0}} \oplus x(1)^{\oplus m_{1}} \oplus \cdots \oplus x(N)^{\oplus m_{N}}
$$

by irreducible representations $x(i)$, and furthermore such a decomposition is unique up to permutation on $\{0,1, \ldots, N\}$.

Since the vector space at the vertex $\infty$ of the representation $x$ is just $\mathbb{C}$, we may assume that the vector space at $\infty$ of $x(0)$ is nonzero and those of all the other $x(i)$ are zero. Then we must have $m_{0}=1$ and the vector space at $\infty$ of $x(0)$ is just $\mathbb{C}$. Now for each $i \geq 0$, the vector space $W(i)$ is given by the direct sum of those of $x(i)$ among all the vertices contained in $D$.

Lemma 3.7. A datum $(V, W, T, Q, P)$ with $V \neq 0$ is stable if and only if the following two conditions hold for any $t \in D$ :

(a) $\operatorname{Ker} Q_{t} \cap \operatorname{Ker} N_{t}=0$;

(b) $\operatorname{Im} P_{t}+\operatorname{Im} N_{t}=W_{t}$ 
Proof. Without loss of generality we may assume $D=\{0\}$, and hence $T \equiv N$ is nilpotent. We drop the subscript $t=0$ in what follows.

The 'only if' part is obvious because $(0, \operatorname{Ker} Q \cap \operatorname{Ker} N)$ and $(V, \operatorname{Im} P+\operatorname{Im} N)$ are both subrepresentations. We show the 'if' part. Suppose that an $N$-invariant subspace $Y \subset W$ is contained in $\operatorname{Ker} Q$ (i.e. $(0, Y)$ is a subrepresentation). If $Y$ is nonzero, then we can take a nonzero vector $w \in Y$ satisfying $N w=0$ because $N$ is nilpotent. Thus $w \in \operatorname{Ker} N \cap Y \subset \operatorname{Ker} N \cap \operatorname{Ker} Q$, which is a contradiction. Hence $Y=0$. Next suppose that an $N$-invariant subspace $Y \subset W$ contains $\operatorname{Im} P$ (i.e. $(V, Y)$ is a subrepresentation). If $Y \neq W$, then consider the endomorphism $N_{Y}$ on $W / Y$ induced from $N$. Since $N_{Y}$ is nilpotent and $W / Y$ is nonzero, Coker $N_{Y}$ is nonzero. Thus we can take a vector $w \in W$ such that $w \notin Y+\operatorname{Im} N \supset \operatorname{Im} P+\operatorname{Im} N$, which is a contradiction. Hence $Y=W$.

\section{Properties of the map $\Phi_{T}$}

Proposition 4.1. Suppose that two data $(V, W, T, Q, P)$ and $\left(V, W^{\prime}, T^{\prime}, Q^{\prime}, P^{\prime}\right)$ with the same $V \neq 0$ are both stable and $\Phi_{T}(Q, P)=\Phi_{T^{\prime}}\left(Q^{\prime}, P^{\prime}\right)$. Then there exists an isomorphism $f: W \rightarrow W^{\prime}$ such that

$$
Q^{\prime}=Q f^{-1}, \quad P^{\prime}=f P, \quad T^{\prime}=f T f^{-1} .
$$

Proof. For each $t \in D$, set $\widehat{W}_{t}:=W_{t} \oplus W_{t}^{\prime}$ and define

$$
\begin{aligned}
& \left(\widehat{Q}_{t}, \widehat{P}_{t}\right):=\left(Q_{t}, P_{t}\right) \oplus(0,0) \in \mathbf{M}\left(V, \widehat{W}_{t}\right)=\mathbf{M}\left(V, W_{t}\right) \oplus \mathbf{M}\left(V, W_{t}^{\prime}\right), \\
& \left(\widehat{Q}_{t}^{\prime}, \widehat{P}_{t}^{\prime}\right):=(0,0) \oplus\left(Q_{t}^{\prime}, P_{t}^{\prime}\right) \in \mathbf{M}\left(V, \widehat{W}_{t}\right),
\end{aligned}
$$

and

$$
\widehat{N}_{t}:=\left(\begin{array}{cc}
N_{t} & 0 \\
0 & 0
\end{array}\right), \quad \widehat{N}_{t}^{\prime}:=\left(\begin{array}{cc}
0 & 0 \\
0 & N_{t}^{\prime}
\end{array}\right) \in \operatorname{End}\left(\widehat{W}_{t}\right) .
$$

Then $\left(\widehat{Q}_{t}, \widehat{P}_{t}, \widehat{N}_{t}\right)_{t \in D}$ and $\left(\widehat{Q}_{t}^{\prime}, \widehat{P}_{t}^{\prime}, \widehat{N}_{t}^{\prime}\right)_{t \in D}$ give points $x, x^{\prime}$ in $\operatorname{Rep}_{Q}(V, \widehat{\mathbf{W}})$ respectively, where $\widehat{\mathbf{W}}=\left(\widehat{W}_{t}\right)_{t \in D}$.

It is known [32, Theorem 1.3] that the ring $\mathbb{C}\left[\operatorname{Rep}_{Q}(V, \widehat{\mathbf{W}})\right]^{\mathrm{GL}(\widehat{\mathbf{W}})}$ of $\mathrm{GL}(\widehat{\mathbf{W}})$-invariant regular functions on $\operatorname{Rep}_{Q}(V, \widehat{\mathbf{W}})$ is generated by the functions

$$
\begin{aligned}
& x=\left(\widehat{Q}_{t}, \widehat{P}_{t}, \widehat{N}_{t}\right)_{t \in D} \longmapsto \chi\left(\widehat{Q}_{s} \widehat{N}_{s}^{k} \widehat{P}_{s}\right), \quad \chi \in \operatorname{End}(V)^{*}, k \geq 0, s \in D, \\
& x=\left(\widehat{Q}_{t}, \widehat{P}_{t}, \widehat{N}_{t}\right)_{t \in D} \longmapsto \operatorname{tr} \widehat{N}_{s}^{k}, \quad k \geq 1, s \in D .
\end{aligned}
$$

Since $\widehat{N}_{t}, \widehat{N}_{t}^{\prime}$ are nilpotent, the hypothesis $\Phi_{T}(Q, P)=\Phi_{T^{\prime}}\left(Q^{\prime}, P^{\prime}\right)$ implies that the two points $x$ and $x^{\prime}$ can not be distinguished by $\operatorname{GL}(\widehat{\mathbf{W}})$-invariant functions, in other words,

$$
\overline{\mathrm{GL}(\widehat{\mathbf{W}}) \cdot x} \cap \overline{\mathrm{GL}(\widehat{\mathbf{W}}) \cdot x^{\prime}} \neq \emptyset
$$


On the other hand, by the construction and Lemma 3.6, the above two orbits are closed. Thus $x$ and $x^{\prime}$ must be isomorphic as representations of $Q_{\operatorname{dim} V}$. The uniqueness of the decomposition in Lemma3.6 implies the result.

Lemma 4.2. For any system $A(z) \in \mathcal{E}_{\vec{k}}(V)$, there exists a datum $(V, W, T, Q, P)$ satisfying $\Phi_{T}(Q, P)=A(z)$.

Proof. Set $\widehat{W}_{t}:=V^{\oplus k_{t}}$ and

$$
\begin{aligned}
& \widehat{Q}_{t}:=\left(\begin{array}{llll}
A_{t, k_{t}} & A_{t, k_{t}-1} & \cdots & A_{t, 1}
\end{array}\right) \in \operatorname{Hom}\left(\widehat{W}_{t}, V\right), \\
& \widehat{P}_{t}:=\left(\begin{array}{c}
0 \\
\vdots \\
0 \\
\operatorname{Id}_{V}
\end{array}\right) \in \operatorname{Hom}\left(V, \widehat{W}_{t}\right), \quad \widehat{N}_{t}:=\left(\begin{array}{cccc}
0 & \operatorname{Id}_{V} & & 0 \\
& 0 & \ddots & \\
& & \ddots & \operatorname{Id}_{V} \\
0 & & & 0
\end{array}\right) \in \operatorname{End}\left(\widehat{W}_{t}\right) \text {. }
\end{aligned}
$$

Then one can easily check

$$
\widehat{Q}_{t} \widehat{N}_{t}^{k} \widehat{P}_{t}=A_{t, k+1}, k=0,1, \ldots, k_{t}-1, \quad \widehat{N}_{t}^{k_{t}}=0 .
$$

Thus setting

$$
\widehat{W}:=\bigoplus \widehat{W}_{t}, \quad \widehat{T}:=\bigoplus\left(t \operatorname{Id}_{W_{t}}+\widehat{N}_{t}\right) \in \operatorname{End}(W),
$$

and $(\widehat{Q}, \widehat{P}):=\bigoplus\left(\widehat{Q}_{t}, \widehat{P}_{t}\right) \in \mathbf{M}(V, \widehat{W})$, we obtain the result.

Remark 4.3. The datum defined above has the following meaning. The identification $\mathbb{C}^{k_{t}} \simeq S_{k_{t}}$ given by the basis $\left\{z^{k_{t}-1}, z^{k_{t}-2}, \ldots, 1\right\}$ induces isomorphisms

$$
\begin{gathered}
\widehat{W}_{t} \simeq \mathbb{C}^{k_{t}} \otimes_{\mathbb{C}} V \simeq S_{k_{t}} \otimes_{\mathbb{C}} V, \\
\operatorname{Hom}_{\mathbb{C}}\left(V, \widehat{W}_{t}\right) \simeq V^{*} \otimes_{\mathbb{C}} V \otimes_{\mathbb{C}} S_{k_{t}} \simeq \operatorname{End}_{\mathbb{C}}(V) \otimes_{\mathbb{C}} S_{k_{t}}, \\
\operatorname{Hom}_{\mathbb{C}}\left(\widehat{W}_{t}, V\right) \simeq \operatorname{End}_{\mathbb{C}}(V) \otimes_{\mathbb{C}} S_{k_{t}}^{*} \simeq \operatorname{End}_{\mathbb{C}}(V) \otimes_{\mathbb{C}} S^{k_{t}}, \\
\operatorname{End}_{\mathbb{C}}\left(\widehat{W}_{t}\right) \simeq \operatorname{End}_{\mathbb{C}}(V) \otimes_{\mathbb{C}} \operatorname{End}_{\mathbb{C}}\left(S_{k_{t}}\right) .
\end{gathered}
$$

Under these identifications, we can write

$$
\begin{aligned}
& \widehat{Q}_{t}=\sum_{k=1}^{k_{t}} A_{t, k} \otimes_{\mathbb{C}} z^{-k} \in \operatorname{Hom}_{\mathbb{C}}\left(\widehat{W}_{t}, V\right), \\
& \widehat{P}_{t}=\operatorname{Id}_{V} \otimes_{\mathbb{C}} 1 \in \operatorname{Hom}_{\mathbb{C}}\left(V, \widehat{W}_{t}\right), \\
& \widehat{N}_{t}=\operatorname{Id}_{V} \otimes_{\mathbb{C}} z \operatorname{Id}_{S_{k_{t}}} \in \operatorname{End}_{\mathbb{C}}\left(\widehat{W}_{t}\right) .
\end{aligned}
$$

The above description was also used by Woodhouse [42]. Note that looking at $\widehat{N}_{t}$ in particular, we have

$$
\left\{L \in \operatorname{End}_{\mathbb{C}}\left(\widehat{W}_{t}\right) \mid L \widehat{N}_{t}=\widehat{N}_{t} L\right\}=\operatorname{End}_{S_{k_{t}}}\left(\widehat{W}_{t}\right)=\mathfrak{g}_{k_{t}}(V) .
$$


Remark 4.4. For any nilpotent endomorphism $N \in \operatorname{End}(W)$, there exist a decomposition $W=\bigoplus_{k} W_{k}$ and injections $\iota: W_{k} \hookrightarrow W_{k-1}$ such that

$$
N=\left(\begin{array}{cccc}
0 & \iota & & 0 \\
& 0 & \ddots & \\
& & \ddots & \iota \\
0 & & & 0
\end{array}\right) .
$$

Such a normal form was effectively used by Oshima [37, 38].

Proposition 4.5. For any system $A(z) \in \mathcal{E}_{\vec{k}}(V)$ with $V \neq 0$, there exists a stable datum $(V, W, T, Q, P)$ satisfying $\Phi_{T}(Q, P)=A(z)$.

Proof. Take a datum $(V, W, T, Q, P)$ satisfying $\Phi_{T}(Q, P)=A(z)$. We show it is stable if $\operatorname{dim} W$ is minimal among all such data. Assume that it is not stable, so we have a subrepresentation $(X, Y)$ such that:

(a) $X=0$ and $Y \neq 0$, or

(b) $X=V$ and $Y \neq W$.

First assume $X=0$ and $Y \neq 0$. Then $Y$ satisfies $Q(Y)=0$ and $T(Y) \subset Y$. Thus the datum $(V, W, T, Q, P)$ induces a datum $\left(V, W / Y, T^{\prime}, Q^{\prime}, P^{\prime}\right)$ in the obvious way. Clearly we have

$$
Q\left(z \operatorname{Id}_{W}-T\right)^{-1} P=Q^{\prime}\left(z \operatorname{Id}_{W / Y}-T^{\prime}\right)^{-1} P^{\prime}
$$

which contradicts the assumption that $\operatorname{dim} W$ is minimal. Next assume that $X=V$ and $Y \neq W$. Then $Y$ satisfies $\operatorname{Im} P \subset Y$ and $T(Y) \subset Y$. Thus the datum $(V, W, T, Q, P)$ induces a datum $\left(V, Y,\left.T\right|_{Y},\left.Q\right|_{Y}, P\right)$ in the obvious way, and clearly

$$
Q\left(z \operatorname{Id}_{W}-T\right)^{-1} P=\left.Q\right|_{Y}\left(z \operatorname{Id}_{Y}-\left.T\right|_{Y}\right)^{-1} P,
$$

which contradicts the assumption again. Hence $(V, W, T, Q, P)$ is stable.

In fact, for given $A(z) \in \mathcal{E}_{\vec{k}}(V)$, we can also construct explicitly a stable datum satisfying $\Phi_{T}(Q, P)=A(z)$ as follows. Let $(V, \widehat{W}, \widehat{T}, \widehat{Q}, \widehat{P})$ be the datum defined in the proof of Lemma 4.2, and set 2

$$
\widehat{A}_{t}:=\left(\begin{array}{cccc}
A_{t, k_{t}} & A_{t, k_{t}-1} & \cdots & A_{t, 1} \\
& A_{t, k_{t}} & \ddots & \vdots \\
& & \ddots & A_{t, k_{t}-1} \\
0 & & & A_{t, k_{t}}
\end{array}\right) \in \operatorname{End}\left(\widehat{W}_{t}\right) .
$$

\footnotetext{
${ }^{2}$ The definition of the matrix $\widehat{A_{t}}$ was suggested by Takemura.
} 
Then one can easily see

$$
\widehat{A}_{t} \widehat{N}_{t}=\widehat{N}_{t} \widehat{A}_{t}, \quad \widehat{Q}_{t}=\left(\begin{array}{llll}
\operatorname{Id}_{V} & 0 & \cdots & 0
\end{array}\right) \widehat{A}_{t} .
$$

The second relation implies $\operatorname{Ker} \widehat{A_{t}} \subset \operatorname{Ker} \widehat{Q}_{t}$. Thus setting $W^{\prime}:=\bigoplus_{t} \operatorname{Ker} \widehat{A}_{t}$, we see that the pair $\left(0, W^{\prime}\right)$ is a subrepresentation of the datum $(V, \widehat{W}, \widehat{T}, \widehat{Q}, \widehat{P})$. Now let $(V, W, T, Q, P)$ be the quotient datum of $(V, \widehat{W}, \widehat{T}, \widehat{Q}, \widehat{P})$ by $\left(0, W^{\prime}\right)$, namely, $W:=$ $\widehat{W} / W^{\prime}=\bigoplus \widehat{W}_{t} / \operatorname{Ker} \widehat{A}_{t}$ and $T, Q, P$ are the maps induced from $\widehat{T}, \widehat{Q}, \widehat{P}$ respectively.

Definition 4.6. The datum $(V, W, T, Q, P)$ given above is called the canonical datum for the system $A(z) \in \mathcal{E}_{\vec{k}}(V)$.

Proposition 4.7. The canonical datum for any $A(z) \in \mathcal{E}_{\vec{k}}(V)$ with $V \neq 0$ is stable.

Proof. We use Lemma 3.7. The condition $\operatorname{Im} P_{t}+\operatorname{Im} N_{t}=W_{t}$ is equivalent to $\operatorname{Im} \widehat{P}_{t}+$ $\operatorname{Im} \widehat{N}_{t}+\operatorname{Ker} \widehat{A}_{t}=\widehat{W}_{t}$, which immediately follows from the definitions of $\widehat{P}_{t}$ and $\widehat{N}_{t}$. To prove $\operatorname{Ker} Q_{t} \cap \operatorname{Ker} N_{t}=0$, suppose that $\hat{w} \in \widehat{W}_{t}$ represents some element in $\operatorname{Ker} Q_{t} \cap \operatorname{Ker} N_{t}$. Then we have

$$
\widehat{Q}_{t} \hat{w}=0, \quad \widehat{A}_{t} \widehat{N}_{t} \hat{w}=0 .
$$

The first equation implies

$$
\widehat{P}_{t}\left(\operatorname{Id}_{V} \quad 0 \quad \cdots \quad 0\right) \widehat{A}_{t} \hat{w}=\widehat{P}_{t} \widehat{Q}_{t} \hat{w}=0,
$$

and on the other hand, it is easy to see that

$$
\operatorname{det}\left[\widehat{P}_{t}\left(\operatorname{Id}_{V} \quad 0 \quad \cdots \quad 0\right)+\widehat{N}_{t}\right] \neq 0 .
$$

Since $\widehat{N}_{t} \widehat{A}_{t} \hat{w}=\widehat{A}_{t} \widehat{N}_{t} \hat{w}=0$, we obtain $\widehat{A}_{t} \hat{w}=0$. Hence $\operatorname{Ker} Q_{t} \cap \operatorname{Ker} N_{t}=0$.

By Lemma 3.7, the datum is stable.

Remark 4.8. On the viewpoint mentioned in Remark 4.3 , the matrix $\widehat{A_{t}}$ is written as

$$
\widehat{A_{t}}=\sum_{k=1}^{k_{t}} A_{t, k} \otimes_{\mathbb{C}} z^{k_{t}-k} \operatorname{Id}_{S_{k_{t}}} \in \operatorname{End}_{\mathbb{C}}(V) \otimes_{\mathbb{C}} \operatorname{End}_{\mathbb{C}}\left(S_{k_{t}}\right)=\operatorname{End}_{\mathbb{C}}\left(\widehat{W}_{t}\right) .
$$

Remark 4.9. In what follows we assume $D=\{0\}$ and omit the subscript $t=0$.

(a) The matrix $\widehat{A} \in \operatorname{End}(\widehat{W})$ is invertible if and only if the top coefficient $A_{k}$ of $A(z)$ is invertible.

(b) Set $d:=\operatorname{ord}(A)$ (see (2.1) for the definition) and identify $\mathbb{C}^{k}$ with $S_{k}$ as in Remark 4.3. Then it is easy to see that $\widehat{A}$ vanishes on the subspace $V \otimes z^{d} S_{k}$ and so induces a homomorphism

$$
V \otimes S_{d} \simeq V \otimes\left(S_{k} / z^{d} S_{k}\right) \stackrel{\widehat{A}}{\rightarrow} V \otimes\left(S_{k} / z^{d} S_{k}\right) \simeq V \otimes S_{d}
$$


Clearly it coincides with the matrix $\widehat{A} \in \operatorname{End}\left(V \otimes \mathbb{C}^{d}\right)$ constructed from $A(z)$ regarded as an element of $\mathcal{E}_{d}(V)$. Hence the construction of the canonical datum does not depend on the choice of $k \geq \operatorname{ord}(A)$.

(c) For $i=1,2$, let $V^{i}$ be a nonzero finite-dimensional $\mathbb{C}$-vector space, $A^{i}(z) \in$ $\mathcal{E}_{k}\left(V^{i}\right)$, and $\left(V^{i}, W^{i}, N^{i}, Q^{i}, P^{i}\right)$ be the canonical datum for $A^{i}(z)$. Then the canonical datum for the direct sum $A(z):=A^{1}(z) \oplus A^{2}(z) \in \mathcal{E}_{k}\left(V^{1} \oplus V^{2}\right)$ is naturally identified with

$$
\bigoplus_{i=1,2}\left(V^{i}, W^{i}, N^{i}, Q^{i}, P^{i}\right)=\left(V^{1} \oplus V^{2}, W^{1} \oplus W^{2}, N^{1} \oplus N^{2}, Q^{1} \oplus Q^{2}, P^{1} \oplus P^{2}\right) .
$$

More generally, if $\left(V^{i}, W^{i}, N^{i}, Q^{i}, P^{i}\right), i=1,2$ are stable data with $V^{i} \neq 0$, then the above direct sum is also stable and

$$
\Phi_{N^{1} \oplus N^{2}}\left(Q^{1} \oplus Q^{2}, P^{1} \oplus P^{2}\right)=\Phi_{N^{1}}\left(Q^{1}, P^{1}\right) \oplus \Phi_{N^{2}}\left(Q^{2}, P^{2}\right) .
$$

For a subset $X$ of $\mathbf{M}(V, W)$ with $V \neq 0$ and an endomorphism $T \in \operatorname{End}(W)$ satisfying (3.1), we set

$$
X^{T \text {-st }}:=\{(Q, P) \in X \mid(V, W, T, Q, P) \text { is stable }\}
$$

One can easily see that $\mathbf{M}(V, W)^{T \text {-st }}$ is invariant under the action of the centralizer $G_{T}$ of $T$ (Note that $G_{T} \subset \mathrm{GL}(\mathbf{W})$ ).

Proposition 4.10. Suppose that $V \neq 0$ and $T \in \operatorname{End}(W)$ satisfies (3.1).

(a) The $G_{T}$-action on $\mathbf{M}(V, W)^{T \text {-st }}$ is free and proper.

(b) The map $\Phi_{T}$ induces a Poisson embedding

$$
\mathbf{M}(V, W)^{T-\text { st }} / G_{T} \hookrightarrow \mathcal{E}_{\vec{k}}(V) .
$$

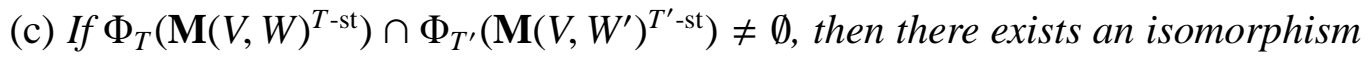
$f: W \rightarrow W^{\prime}$ such that $T^{\prime}=f T f^{-1}$.

(d) The subset $\mathbf{M}(V, W)^{T \text {-st }}$ is invariant under the action of $G_{\vec{k}}(V)$.

Proof. (a) Considering the $G_{T}$-equivariant closed embedding

$$
\varphi: \mathbf{M}(V, W) \hookrightarrow \operatorname{Rep}_{Q}(V, \mathbf{W}), \quad(Q, P) \mapsto\left(Q_{t}, P_{t}, N_{t}\right)_{t \in D},
$$

we see that the action is free by Lemma 3.5. To see properness, we use an identification $\operatorname{Rep}_{Q}(V, \mathbf{W}) \simeq \operatorname{Rep}_{Q_{\operatorname{dim} V}}(\mathbb{C}, \mathbf{W})$ and consider the subset of irreducible representations $\operatorname{Rep}_{Q_{\operatorname{dim} V}}^{\text {irr }}(\mathbb{C}, \mathbf{W})$. We have

$$
\varphi\left(\mathbf{M}(V, W)^{T \text {-st }}\right)=\varphi(\mathbf{M}(V, W)) \cap \operatorname{Rep}_{Q_{\operatorname{dim} V}}^{\operatorname{irr}}(\mathbb{C}, \mathbf{W}) .
$$


By a standard fact in the geometric invariant theory [33, Corollary 2.5] together with King's work [29], the $\operatorname{GL}(\mathbf{W})$-action on $\operatorname{Rep}_{Q_{\operatorname{dim} V}}^{\operatorname{irr}}(\mathbb{C}, \mathbf{W})$ is proper. Therefore the above implies the properness of the $G_{T}$-action on $\mathbf{M}(V, W)^{T \text {-st }}$.

(b) and (c) follow from Proposition 4.1 .

(d) The explicit description of the action (2.2) shows that

$$
\begin{aligned}
\operatorname{Ker}(g \cdot Q)_{t} \cap \operatorname{Ker} N_{t} & =\operatorname{Ker} Q_{t} \cap \operatorname{Ker} N_{t} \\
\operatorname{Im}(g \cdot P)_{t}+\operatorname{Im} N_{t} & =\operatorname{Im} P_{t}+\operatorname{Im} N_{t}
\end{aligned}
$$

for any $(Q, P) \in \mathbf{M}(V, W), g \in G_{\vec{k}}(V)$ and $t \in D$. Therefore Lemma 3.7 implies the result.

Let $\mathfrak{g}_{T}$ be the Lie algebra of $G_{T}$ and $p_{T}: \mathfrak{g l}(W) \rightarrow \mathfrak{g}_{T}^{*}$ be the transpose of the inclusion $\mathfrak{g}_{T} \hookrightarrow \mathfrak{g l}(W)$. Recall that the map

$$
\mu_{W}: \mathbf{M}(V, W) \rightarrow \mathfrak{g l}(W) ; \quad(Q, P) \mapsto-P Q
$$

is a moment map generating the $\operatorname{GL}(W)$-action. We set

$$
\mu_{T}:=p_{T} \circ \mu_{W}: \mathbf{M}(V, W) \rightarrow \mathfrak{g}_{T}^{*},
$$

which is a $G_{T}$-moment map.

Lemma 4.11. The map $\mu_{T}$ is $G_{\vec{k}}(V)$-invariant.

Proof. For each $t \in D$, let $G_{N_{t}} \subset \mathrm{GL}\left(W_{t}\right)$ be the centralizer of $N_{t}$, $\mathfrak{g}_{N_{t}}$ be its Lie algebra, and $p_{t}: \mathfrak{g l}\left(W_{t}\right) \rightarrow \mathfrak{g}_{N_{t}}^{*}$ be the transpose of the inclusion $g_{N_{t}} \hookrightarrow \mathfrak{g l}\left(W_{t}\right)$. Then obviously we have $p_{t}\left(A_{t} N_{t}\right)=p_{t}\left(N_{t} A_{t}\right)$ for any $A_{t} \in \mathfrak{g l}\left(W_{t}\right)$, which implies

$$
\begin{aligned}
p_{t}\left(\left(g_{t} \cdot P_{t}\right)\left(g_{t} \cdot Q_{t}\right)\right) & =p_{t}\left(\sum_{k, l \geq 0} N_{t}^{k} P_{t}\left(g_{t}^{-1}\right)_{k} g_{t, l} Q_{t} N_{t}^{l}\right) \\
& =p_{t}\left(\sum_{k, l \geq 0} N_{t}^{k+l} P_{t}\left(g_{t}^{-1}\right)_{k} g_{t, l} Q_{t}\right) .
\end{aligned}
$$

Substituting the equality $g_{t}^{-1}(z) g_{t}(z)=1\left(\bmod z^{k_{t}}\right)$ into the above, we have

$$
p_{t}\left(\left(g_{t} \cdot P_{t}\right)\left(g_{t} \cdot Q_{t}\right)\right)=p_{t}\left(P_{t} Q_{t}\right)
$$

Since

$$
\mu_{T}(Q, P)=-\left[p_{t}\left(P_{t} Q_{t}\right)\right]_{t \in D} \in \bigoplus_{t} \mathfrak{g}_{N_{t}}^{*}=\mathfrak{g}_{T}
$$

we obtain the result. 
Lemma 4.12. Suppose $V \neq 0$. Then the tangent space of the $G_{\vec{k}}(V)$-orbit through any $x \in \mathbf{M}(V, W)^{T \text {-st }}$ coincides with $\operatorname{Ker} d_{x} \mu_{T}$, and its dimension is constant on $\mathbf{M}(V, W)^{T \text {-st }}$.

Proof. The moment map equations for $\Phi_{T}$ and $\mu_{T}$ imply

$$
\operatorname{Ker} d_{x} \mu_{T}=T_{x}\left(G_{T} \cdot x\right)^{\omega}, \quad \operatorname{Ker} d_{x} \Phi_{T}=T_{x}\left(G_{\vec{k}}(V) \cdot x\right)^{\omega}
$$

where the superscript $\omega$ means the symplectic orthogonal complement subspace. On the other hand, Proposition 4.10, (b) implies

$$
\operatorname{Ker} d_{x} \Phi_{T}=T_{x}\left(G_{T} \cdot x\right)
$$

Hence

$$
\operatorname{Ker} d_{x} \mu_{T}=T_{x}\left(G_{T} \cdot x\right)^{\omega}=\left(\operatorname{Ker} d_{x} \Phi_{T}\right)^{\omega}=T_{x}\left(G_{\vec{k}}(V) \cdot x\right),
$$

whose dimension is constant on $\mathbf{M}(V, W)^{T \text {-st }}$ by Proposition 4.10, (a).

Remark 4.13. The above lemma implies that for any $x \in \mathbf{M}(V, W)^{T \text {-st }}$, the subspaces $\operatorname{Ker} d_{x} \mu_{T}$ and $\operatorname{Ker} d_{x} \Phi_{T}$ are symplectic orthogonal complement to each other, namely the pair $\left(\mu_{T}, \Phi_{T}\right)$ is a dual pair of moment maps in the sense of Weinstein [41], as mentioned by Harnad [21].

Theorem 4.14. Suppose $V \neq 0$. Then for any $G_{\vec{k}}(V)$-coadjoint orbit $\mathbb{O}$, there exist a finite-dimensional $\mathbb{C}$-vector space $W$, an endomorphism $T$ of $W$, and a $G_{T}$-coadjoint orbit $O \subset \mathfrak{g}_{T}^{*}$ such that the map $\Phi_{T}$ induces a symplectomorphism

$$
\mu_{T}^{-1}(O)^{T-\mathrm{st}} / G_{T} \stackrel{\simeq}{\rightarrow} \mathbb{O} .
$$

Moreover the choice of $(W, T, O)$ is unique in the following sense: if another triple $\left(W^{\prime}, T^{\prime}, O^{\prime}\right)$ has the same properties, then there exists an isomorphism $f: W \rightarrow W^{\prime}$ such that $f T f^{-1}=T^{\prime}$ and $f O f^{-1}=O^{\prime}$.

Proof. Take any $A(z) \in \mathbb{O}$ and then a stable datum $(V, W, T, Q, P)$ satisfying $A(z)=$ $\Phi_{T}(Q, P)$. Let $O \subset \mathfrak{g}_{T}^{*}$ be the coadjoint orbit through $\mu_{T}(Q, P)$. Then by Proposition 4.10, (d) and Lemma 4.11, the subset $\mu_{T}^{-1}(O)^{T \text {-st }}$ is $G_{\vec{k}}(V)$-invariant and hence by equivariance, its image under $\Phi_{T}$ is a $G_{\vec{k}}(V)$-invariant subset of $\mathfrak{g}_{\vec{k}}^{*}(V)$ containing $\mathbb{O}$. To see that the image actually coincides with $\mathbb{O}$, we have to show that the induced $G_{\vec{k}}(V)$ action on $\mu_{T}^{-1}(O)^{T \text {-st }} / G_{T}$ is transitive, or equivalently, the restricted $G_{\vec{k}}(V)$-action on $\mu_{T}^{-1}(\alpha)^{T \text {-st }}$, where $\alpha \in O$, is transitive.

Proposition 4.10, (a) implies that $\mu_{T}^{-1}(\alpha)^{T \text {-st }}$ is a pure-dimensional smooth subvariety, and in which, by Lemma 4.12, any $G_{\vec{k}}(V)$-orbit is a Zariski open subset. Therefore 
in order to show the transitivity of the action, it is sufficient to show that $\mu_{T}^{-1}(\alpha)^{T \text {-st }}$ is an irreducible variety.

To see that $\mu_{T}^{-1}(\alpha)^{T \text {-st }}$ is irreducible, consider the first projection $\varphi: \mu_{T}^{-1}(\alpha)^{T \text {-st }} \rightarrow$ $\operatorname{Hom}(W, V)$. By the definition of $\mu_{T}$ and Lemma 3.7, we see that any nonempty fiber of $\varphi$ is a Zariski open subset of an affine space. Moreover Lemma 4.15 below shows that $\varphi$ is a dominant morphism and every nonempty fibers have the same dimension, because any $g \in G_{\vec{k}}(V)$ induces an isomorphism between the fibers $\varphi^{-1}(Q)$ and $\varphi^{-1}(g$. $Q$ ). Therefore we may apply the following fact (c.f. [14, Lemma 6.1]) to deduce that $\mu_{T}^{-1}(\alpha)^{T \text {-st }}$ is irreducible:

If $X$ is a pure-dimensional scheme, $Y$ is an irreducible scheme and $f: X \rightarrow Y$ is a dominant morphism with all fibers irreducible of constant dimension, then $X$ is irreducible.

The uniqueness assertion immediately follows from Proposition 4.1 .

Lemma 4.15. Let $V, W$ be two $\mathbb{C}$-vector spaces and let $N \in \operatorname{End}(W)$ be a nilpotent endomorphism of $W$ with $N^{k}=0$. Define an action of $G_{k}(V)$ on $\operatorname{Hom}(W, V)$ as in (2.2). Then the restricted action on the subset

$$
\left\{Q \in \operatorname{Hom}(W, V)|Q|_{\text {Ker } N} \text { is injective }\right\}
$$

is transitive.

Proof. Let $Q, Q^{\prime} \in \operatorname{Hom}(W, V)$ and assume that both $\left.Q\right|_{\text {Ker } N}$ and $\left.Q^{\prime}\right|_{\text {Ker } N}$ are injective. Then we solve the equation

$$
\sum_{i=0}^{k-1} g_{i} Q N^{i}=Q^{\prime}, \quad g(z)=\sum_{i} g_{i} z^{i} \in G_{k}(V) .
$$

First we restrict the both sides to $\operatorname{Ker} N$. Then we have

$$
\left.g_{0} Q\right|_{\text {Ker } N}=\left.Q^{\prime}\right|_{\text {Ker } N}
$$

By the assumption we can find $g_{0} \in \mathrm{GL}(V)$ satisfying the above. Next, we restrict the both sides of (4.3) to $\operatorname{Ker} N^{2}$. Then we have

$$
\left.\left(g_{0} Q+g_{1} Q N\right)\right|_{\operatorname{Ker} N^{2}}=\left.Q^{\prime}\right|_{\operatorname{Ker} N^{2}}
$$

or equivalently,

$$
\left.g_{1} Q N\right|_{\text {Ker } N^{2}}=\left.\left(Q^{\prime}-g_{0} Q\right)\right|_{\operatorname{Ker} N^{2}} .
$$


Because $\left.Q\right|_{\text {Ker } N}$ is injective, the kernel of $\left.Q N\right|_{\operatorname{Ker} N^{2}}$ is just $\operatorname{Ker} N$. Also, we have $Q^{\prime}-$ $\left.g_{0} Q\right|_{\text {Ker } N}=0$. Hence the both sides of the above equation descend to homomorphisms from $\operatorname{Ker} N^{2} / \operatorname{Ker} N$;

$$
\left.g_{1} Q N\right|_{\operatorname{Ker} N^{2} / \operatorname{Ker} N}=\left.\left(Q^{\prime}-g_{0} Q\right)\right|_{\operatorname{Ker} N^{2} / \operatorname{Ker} N}
$$

and we can find $g_{1} \in \operatorname{End}(W)$ satisfying the above as $\left.Q N\right|_{\operatorname{Ker} N^{2} / \operatorname{Ker} N}$ is injective.

Iterating this argument inductively, we find $g_{i} \in \operatorname{End}(W), i=0,1, \ldots, k-1$ with $\operatorname{det} g_{0} \neq 0$, satisfying the equation

$$
\left.g_{i} Q N^{i}\right|_{\operatorname{Ker} N^{i+1}}=\left.\left(Q^{\prime}-\sum_{j=0}^{i-1} g_{j} Q N^{j}\right)\right|_{\operatorname{Ker} N^{i+1}},
$$

and finally we obtain a desired $g(z) \in G_{k}(V)$.

\section{Harnad duality}

In this section we formulate the 'Harnad dual' in a categorical setting, and introduce some important properties of it.

Definition 5.1. We call a sextuple $(V, W, S, T, Q, P)$ consisting of:

- two finite-dimensional $\mathbb{C}$-vector spaces $V, W$;

- $(S, T) \in \operatorname{End}(V) \oplus \operatorname{End}(W)$; and

- $(Q, P) \in \mathbf{M}(V, W)$,

as a Harnad datum.

We always assume our Harnad data $(V, W, S, T, Q, P)$ satisfy Condition (3.1) and

$$
\prod_{s \in E}\left(S-s \operatorname{Id}_{V}\right)^{l_{s}}=0
$$

where the zero datum $(0,0,0,0,0,0)$ is understood to satisfy these two conditions.

Harnad data $(V, W, S, T, Q, P)$ (satisfying (3.1) and (5.1) form an abelian category, which we denote by $\vec{H}_{\vec{k}}$. A morphism from $(V, W, S, T, Q, P)$ to $\left(V^{\prime}, W^{\prime}, S^{\prime}, T^{\prime}, Q^{\prime}, P^{\prime}\right)$ is a pair $\left(f_{V}, f_{W}\right)$ of linear maps $f_{V}: V \rightarrow V^{\prime}$ and $f_{W}: W \rightarrow W^{\prime}$ satisfying

$$
\begin{aligned}
& f_{V} S=S^{\prime} f_{V}, \quad f_{W} T=T^{\prime} f_{W}, \\
& f_{V} Q=Q^{\prime} f_{W}, \quad f_{W} P=P^{\prime} f_{V} .
\end{aligned}
$$


Now consider systems of linear ordinary differential equations of the form

$$
\frac{d u}{d z}=A(z) u, \quad A(z)=S+\sum_{t \in D} \sum_{k=1}^{k_{t}} \frac{A_{t, k}}{(z-t)^{k}} \in \mathfrak{g l}(V) \oplus \mathcal{E}_{\vec{k}}(V),
$$

such that $S=\lim _{z \rightarrow \infty} A(z)$ satisfies (5.1). We define an abelian category $\vec{\imath} \mathcal{D}_{\vec{k}}$ by

- an object of $\vec{l}_{\vec{k}}$ is a pair $(V, A)$ consisting of a finite-dimensional $\mathbb{C}$-vector space $V$ and a system $A(z) \in \mathfrak{g l}(V) \oplus \mathcal{E}_{\vec{k}}(V)$ such that $\lim _{z \rightarrow \infty} A(z)$ satisfies (5.1);

- a morphism from $(V, A)$ to $\left(V^{\prime}, A^{\prime}\right)$, where $A(z)=S+\sum A_{t, k}(z-t)^{-k}, A^{\prime}(z)=$ $S^{\prime}+\sum A_{t, k}^{\prime}(z-t)^{-k}$, is a linear map $f: V \rightarrow V^{\prime}$ satisfying $f \circ S=S^{\prime} \circ f$ and $f \circ A_{t, k}=A_{t, k}^{\prime} \circ f$ for all $t, k$.

Then it is easy to see that

$$
\Phi:(V, W, S, T, Q, P) \mapsto\left(V, S+\Phi_{T}(Q, P)\right)=\left(V, S+Q\left(z \operatorname{Id}_{W}-T\right)^{-1} P\right)
$$

defines a functor from ${ }_{\vec{l}} \mathcal{H}_{\vec{k}}$ to $\vec{l} \mathcal{D}_{\vec{k}}$ (the map between the sets of morphisms is given by the projection $\left.\left(f_{V}, f_{W}\right) \mapsto f_{V}\right)$. The notion of canonical datum (Definition 4.6) gives a 'section' of $\Phi$; for $(V, A) \in{ }_{\vec{l}} \mathcal{D}_{\vec{k}}$, letting $S:=\lim _{z \rightarrow \infty} A(z)$ and $(V, W, T, Q, P)$ be the canonical datum for the $\mathcal{E}_{\vec{k}}(V)$-component of $A(z)$, set

$$
\kappa(V, A):=(V, W, S, T, Q, P)
$$

By the construction of the canonical datum, it then defines a functor from ${ }_{\vec{l}} \mathcal{D}_{\vec{k}}$ to ${ }_{\vec{l}} \mathcal{H}_{\vec{k}}$ and satisfies $\Phi \circ \kappa=$ Id. Note that there is an equivalence of categories

$$
\sigma:{ }_{\vec{l}} \mathcal{H}_{\vec{k}} \rightarrow{ }_{\vec{k}} \mathcal{H}_{\vec{l}} ; \quad(V, W, S, T, Q, P) \mapsto(W, V, T, S, P, Q),
$$

which together with $\Phi$ induces

$$
\begin{aligned}
\Phi \circ \sigma(V, W, S, T, Q, P) & =\left(W, T+P\left(\zeta \operatorname{Id}_{V}-S\right)^{-1} Q\right) \\
& =\left(W, T-\Psi_{S}(Q, P)\right),
\end{aligned}
$$

where $\zeta$ denotes the indeterminate for $\vec{k} \mathcal{D}_{\vec{l}}$.

Definition 5.2. We call

$$
\mathrm{HD}:=\Phi \circ \sigma \circ \kappa: \vec{k} \mathcal{D}_{\vec{l}} \rightarrow{ }_{\vec{l}} \mathcal{D}_{\vec{k}}
$$

as the Harnad dual functor.

Here we give two simple examples. 
Example 5.3. (a) If any $\mathcal{E}_{k_{t}}(V)$-component of $A(z)$ is zero (i.e., $A(z)=S$ ), then the corresponding canonical datum is zero, whence $\operatorname{HD}(V, S)=(0,0)$.

(b) Let us compute the Harnad dual $\operatorname{HD}(V, A)$ in the case $(V, A)=(\mathbb{C}, s+\alpha)$ for some $s \in E, \alpha(z)=\sum \alpha_{t, j}(z-t)^{-j} \in \mathcal{E}_{\vec{k}}(\mathbb{C})$. Set $d_{t}:=\operatorname{ord}\left(\alpha_{t}\right)$ and let $(\mathbb{C}, W, T, Q, P)$ be the canonical datum for $\alpha(z)$, which defines $\left(W_{t}, N_{t}, Q_{t}, P_{t}\right)$ for each $t \in D$ as usual. Then Remark 4.9 shows that $W_{t}=\mathbb{C}^{d_{t}}$ (which is understood as zero if $d_{t}=0$ ) and

$$
\begin{aligned}
& Q_{t}=\left(\begin{array}{llll}
\alpha_{t, d_{t}} & \alpha_{t, d_{t}-1} & \cdots & \alpha_{t, 1}
\end{array}\right) \in \operatorname{Hom}\left(\mathbb{C}^{d_{t}}, \mathbb{C}\right), \\
& P_{t}=\left(\begin{array}{c}
0 \\
\vdots \\
0 \\
1
\end{array}\right) \in \operatorname{Hom}\left(\mathbb{C}, \mathbb{C}^{d_{t}}\right), \quad N_{t}=\left(\begin{array}{cccc}
0 & 1 & & 0 \\
& 0 & \ddots & \\
& & \ddots & 1 \\
0 & & & 0
\end{array}\right) \in \operatorname{End}\left(\mathbb{C}^{d_{t}}\right) \text {. }
\end{aligned}
$$

For $t, t^{\prime} \in D$, let

$$
R_{t^{\prime}, t}:=P_{t^{\prime}} Q_{t}=\left(\begin{array}{cccc}
0 & 0 & \cdots & 0 \\
\vdots & \vdots & & \vdots \\
0 & 0 & \cdots & 0 \\
\alpha_{t, d_{t}} & \alpha_{t, d_{t}-1} & \cdots & \alpha_{t, 1}
\end{array}\right) \in \operatorname{Hom}\left(\mathbb{C}^{d_{t}}, \mathbb{C}^{d_{t^{\prime}}}\right) .
$$

Then the Harnad dual $(W, B)=\operatorname{HD}(\mathbb{C}, s+\alpha)$ is described as

$$
B(\zeta)=T+\frac{R}{\zeta-s}, \quad R=P Q=\left(R_{t^{\prime}, t}\right)_{t^{\prime}, t \in D}
$$

Considering the sub-objects, we define the following:

Definition 5.4. (a) A subrepresentation of a $\operatorname{Harnad}$ datum $(V, W, S, T, Q, P)$ is a pair $(X, Y)$ of subspaces $X \subset V, Y \subset W$ satisfying

$$
S(X) \subset X, \quad T(Y) \subset Y, \quad Q(Y) \subset X, \quad P(X) \subset Y .
$$

A nonzero Harnad datum $(V, W, S, T, Q, P)$ is said to be irreducible if it has no nonzero proper subrepresentations.

(b) A pair $(V, A) \in{ }_{\vec{l}} \mathcal{D}_{\vec{k}}$ with $V \neq 0$ is said to be irreducible if $V$ has no nonzero proper subspace preserved by all $A_{t, k}$ and $S$.

Any subrepresentation of a Harnad datum $(V, W, S, T, Q, P)$ is also that of the da$\operatorname{tum}(V, W, T, Q, P)$.

Lemma 5.5. If a Harnad datum $(V, W, S, T, Q, P) \in{ }_{\vec{l}} \mathcal{H}_{\vec{k}}$ is irreducible and $V \neq 0$, then the datum $(V, W, T, Q, P)$ is stable. 
Proof. Suppose that a subrepresentation $(X, Y)$ of the datum $(V, W, T, Q, P)$ satisfies $X=0$ or $X=V$. Then $S(X) \subset X$, and hence the pair $(X, Y)$ is a subrepresentation of $(V, W, S, T, Q, P)$. Therefore the irreducibility of $(V, W, S, T, Q, P)$ implies the stability of $(V, W, T, Q, P)$.

Lemma 5.6. Let $(V, W, S, T, Q, P) \in \in_{\vec{l}} \mathcal{H}_{\vec{k}}$ with $V \neq 0$ and suppose that $(V, W, T, Q, P)$ is stable. Then $(V, W, S, T, Q, P)$ is irreducible if and only if $\Phi(V, W, S, T, Q, P) \in{ }_{\vec{l}} \mathcal{D}_{\vec{k}}$ is irreducible. In particular, a pair $(V, A) \in{ }_{\vec{l}} \mathcal{D}_{\vec{k}}$ with $V \neq 0$ is irreducible if and only if $\kappa(V, A) \in{ }_{\vec{l}} \mathcal{H}_{\vec{k}}$ is irreducible.

Proof. We use the normal form mentioned in Remark 4.4 namely, take decompositions $W_{t}=\bigoplus_{k} W_{t, k}$ together with injections $\iota: W_{t, k} \rightarrow W_{t, k-1}$ such that $N_{t}$ is written as in (4.1). We regard $W_{t, k} \subset W_{t, k-1}$ using $\iota$. Note that if we denote by $Q_{t, k}$ (resp. $P_{t, k}$ ) the block components of $Q_{t}\left(\right.$ resp. $\left.P_{t}\right)$ with respect to the decomposition $W_{t}=\bigoplus_{k} W_{t, k}$, the system $A(z):=S+\Phi_{T}(Q, P)$ satisfies

$$
A_{t, k}=\sum_{j \geq 1} Q_{t, j} P_{t, j+k-1} .
$$

Now we show the 'only if' part. Suppose that a subspace $X \subset V$ is preserved by all $A_{t, k}$ and $S$. Then we set

$$
Y_{t, k}:=\sum_{j \geq k} P_{t, j}(X) \subset W_{t, k}, \quad Y_{t}:=\bigoplus_{k} Y_{t, k} \subset W_{t} .
$$

Obviously we have $P_{t}(X) \subset Y_{t}$ and $N_{t}\left(Y_{t}\right) \subset Y_{t}$. Moreover, we have

$$
\begin{aligned}
Q_{t}\left(Y_{t}\right) & =\sum_{k \geq 1} Q_{t, k}\left(Y_{t, k}\right) \\
& =\sum_{k \geq 1} \sum_{j \geq k} Q_{t, k} P_{t, j}(X) \\
& =\sum_{k \geq 1} A_{t, k}(X) \subset X .
\end{aligned}
$$

Thus we see that the pair $(X, Y)$, where $Y=\bigoplus Y_{t}$, is a subrepresentation of the Harnad datum $(V, W, S, T, Q, P)$. Because $(V, W, S, T, Q, P)$ is irreducible, we have $X=0$ or $X=V$. Hence $(V, A)$ is irreducible.

Next we show the 'if' part. Suppose that a subrepresentation $(X, Y)$ of the Harnad datum $(V, W, S, T, Q, P)$ is given. Then we have $S(X) \subset X$ and

$$
A_{t, k}(X)=Q_{t} N_{t}^{k-1} P_{t}(X) \subset X .
$$

Thus by the irreducibility of $(V, A)$ we get $X=0$ or $X=V$. Because the pair $(X, Y)$ is also a subrepresentation of the datum $(V, W, T, Q, P)$ which is stable, this implies $Y=0$ or $Y=W$ respectively. Hence $(V, W, S, T, Q, P)$ is irreducible. 
Lemma 5.7. Let $(V, W, S, T, Q, P) \in{ }_{\vec{l}} \mathcal{H}_{\vec{k}}$ with $V \neq 0$ and suppose that $(V, W, T, Q, P)$ is stable. Then $(V, W, S, T, Q, P)$ and its image under $\kappa \circ \Phi$ are isomorphic as objects in $\vec{l} \mathcal{H}_{\vec{k}}$

$$
\kappa \circ \Phi(V, W, S, T, Q, P) \sim(V, W, S, T, Q, P)
$$

Proof. Note that $\kappa \circ \Phi$ effects no change in both $V$ and $S$. Therefore Proposition 4.1 together with Proposition 4.7 gives a desired isomorphism of the form $\left(\operatorname{Id}_{V}, f\right)$.

Theorem 5.8. If $(V, A) \in{ }_{\vec{l}} \mathcal{D}_{\vec{k}}$ is irreducible and $(V, A) \nsim(\mathbb{C}, s)$ for any $s \in E$, then $\mathrm{HD}(V, A)$ is also irreducible and

$$
\operatorname{HD} \circ \operatorname{HD}(V, A) \sim(V, A) .
$$

Proof. The assumption together with Lemma 5.6 implies that $\kappa(V, A) \in{ }_{\vec{l}} \mathcal{H}_{\vec{k}}$ is irreducible, and so is $\sigma \circ \kappa(V, A)$ since $\sigma$ clearly preserves the irreducibility.

Now set $(V, W, S, T, Q, P):=\kappa(V, A)$. If $W \neq 0$, applying Lemma5.5 to $\sigma \circ \kappa(V, A)$ shows that $(W, V, S, P, Q)$ is stable. Thus we see from Lemma 5.6 that $\operatorname{HD}(V, A)=$ $\Phi \circ \sigma \circ \kappa(V, A)$ is irreducible, and hence by Lemma5.7 we have

$$
\begin{aligned}
\mathrm{HD} \circ \mathrm{HD}(V, A) & =\Phi \circ \sigma \circ(\kappa \circ \Phi) \circ \sigma \circ \kappa(V, A) \\
& \sim \Phi \circ(\sigma \circ \sigma) \circ \kappa(V, A) \\
& =\Phi \circ \kappa(V, A)=(V, A) .
\end{aligned}
$$

If $W=0$, the construction of the canonical datum shows that $A(z)=S$. Then the irreducibility of $(V, A)=(V, S)$ implies that $V=\mathbb{C}$ and $S$ is a scalar satisfying (5.1).

The following is an immediate consequence of the above theorem:

Corollary 5.9. An irreducible pair $(V, A) \in \in_{\vec{l}} \mathcal{D}_{\vec{k}}$ satisfies $\operatorname{HD}(V, A)=(0,0)$ if and only if $(V, A) \sim(\mathbb{C}, s)$ for some $s \in E$.

For a subset $X$ of $\mathbf{M}(V, W)$ with $W \neq 0$ and an endomorphism $S \in \operatorname{End}(V)$ satisfying (5.1), we set

$$
X_{S \text {-st }}:=\{(Q, P) \in \mathbf{M}(V, W) \mid(W, V, S, P, Q) \text { is stable }\} .
$$

Let $G_{S} \subset \mathrm{GL}(V)$ be the centralizer of $S, \mathfrak{g}_{S}$ be its Lie algebra, and $p_{S}: \mathfrak{g l}(V) \rightarrow$ $\mathfrak{g}_{S}^{*}$ be the transpose of the inclusion $\mathfrak{g}_{S} \hookrightarrow \mathfrak{g l}(V)$. Then the composite $\mu_{S}:=p_{S} \circ$ $\mu_{V}: \mathbf{M}(V, W) \rightarrow \mathfrak{g}_{S}^{*}$ is a moment map generating the $G_{S}$-action. 
Theorem 5.10. Let $(V, W, S, T, Q, P)$ be an irreducible Harnad datum with $V, W \neq 0$. Let

- $\mathbb{O}_{V}$ be the $G_{\vec{k}}(V)$-coadjoint orbit through $\Phi_{T}(Q, P)$;

- $\mathbb{O}_{W}$ be the $G_{\vec{l}}(W)$-coadjoint orbit through $\Psi_{S}(Q, P)$;

- $O_{T}$ be the $G_{T}$-coadjoint orbit through $\mu_{T}(Q, P)$; and

- $O_{S}$ be the $G_{S}$-coadjoint orbit through $\mu_{S}(Q, P)$.

Then the two spaces

$$
\mathfrak{M}_{S}^{\mathrm{irr}}\left(\mathbb{O}_{V}, \mathcal{O}_{S}\right):=\left\{\begin{array}{l|l}
A(z) \in S+\mathbb{O}_{V} & \begin{array}{l}
(V, A) \text { is irreducible, } \\
p_{S}\left(\operatorname{Res}_{z=\infty} A(z)\right) \in-O_{S}
\end{array}
\end{array}\right\} / G_{S},
$$

and

$$
\mathfrak{M}_{-T}^{\mathrm{irr}}\left(\mathbb{O}_{W}, \mathcal{O}_{T}\right):=\left\{\begin{array}{l|l}
B(\zeta) \in-T+\mathbb{O}_{W} & \begin{array}{l}
(W, B) \text { is irreducible, } \\
p_{T}\left(\operatorname{Res}_{\zeta=\infty} B(\zeta)\right) \in-\mathcal{O}_{T}
\end{array}
\end{array}\right\} / G_{T},
$$

are both holomorphic symplectic manifolds and symplectomorphic to each other. The symplectomorphism is given by $(W,-B(\zeta)) \sim \mathrm{HD}(V, A(z))$.

Proof. By Theorem 4.14, we have a $G_{S}$-equivariant symplectomorphism

$$
S+\Phi_{T}: \mu_{T}^{-1}\left(\mathcal{O}_{T}\right)^{T-\text { st }} / G_{T} \stackrel{\simeq}{\rightarrow} S+\mathbb{O}_{V} .
$$

Under this isomorphism, the $G_{S}$-moment map $p_{S} \circ \mu_{V}$ on the left hand side corresponds to the map $-p_{S} \circ \operatorname{Res}_{z=\infty}$. Thus we have a bijection

$$
\begin{aligned}
S+\Phi_{T}: \mu_{S}^{-1}\left(O_{S}\right) \cap \mu_{T}^{-1}\left(O_{T}\right)^{T \text {-st }} / G_{S} & \times G_{T} \\
& \rightarrow\left\{A(z) \in S+\mathbb{O}_{V} \mid p_{S}\left(\operatorname{Res}_{z=\infty} A(z)\right) \in-O_{S}\right\} / G_{S} .
\end{aligned}
$$

Similarly, we have a bijection

$$
\begin{aligned}
-T+\Psi_{S}: \mu_{S}^{-1}\left(O_{S}\right)_{S \text {-st }} \cap \mu_{T}^{-1}\left(O_{T}\right) / G_{S} \times G_{T} \\
\rightarrow\left\{B(\zeta) \in-T+\mathbb{O}_{W} \mid p_{T}\left(\operatorname{Res}_{\zeta=\infty} B(\zeta)\right) \in-O_{T}\right\} / G_{T} .
\end{aligned}
$$

If a system $A(z) \in S+\mathbb{O}_{V}$ is irreducible and $(V, W, T, \tilde{Q}, \tilde{P})$ is a stable datum satisfying $\Phi_{T}(\tilde{Q}, \tilde{P})=A(z)-S$, then the Harnad datum $(V, W, S, T, \tilde{Q}, \tilde{P})$ is also irreducible by Lemma 5.6, and hence so is $B(\zeta)=-T+\Psi_{S}(\tilde{Q}, \tilde{P})$ by Lemma 5.6 again. The result follows. 
Remark 5.11. In the situation of Theorem 5.10, take an arbitrary $R \in p_{S}^{-1}\left(O_{S}\right)$ and let $\mathbb{O}_{V, \infty}$ be the $G_{2}(V)$-coadjoint orbit through $-S w^{-2}-R w^{-1}$, where we use $w$ as the indeterminate instead of $z$. Note that $\mathbb{O}_{V, \infty}$ does not depend on the choice of $R$. Indeed, for any other $R^{\prime} \in p_{S}^{-1}\left(O_{S}\right)$, we can find $a \in G_{S}$ satisfying

$$
a R a^{-1}-R^{\prime} \in \operatorname{Ker} p_{S}=\operatorname{Im~ad}_{S},
$$

namely, there exists some $b \in \operatorname{End}(V)$ such that $a R a^{-1}=R^{\prime}+[S, b]$. Then setting $g(w):=a+b a w \in G_{2}(V)$, one can easily check that $g \cdot\left(-S w^{-2}-R w^{-1}\right)=-S w^{-2}-$ $R^{\prime} w^{-1}$.

Using $\mathbb{O}_{V, \infty}$, we now have the following description of $\mathfrak{M}_{S}^{\mathrm{irr}}\left(\mathbb{O}_{V}, O_{S}\right)$ :

$$
\begin{aligned}
& \mathfrak{M}_{S}^{\mathrm{irr}}\left(\mathbb{O}_{V}, \mathcal{O}_{S}\right) \simeq \mathfrak{M}^{\mathrm{irr}}\left(\mathbb{O}_{V}, \mathbb{O}_{V, \infty}\right):= \\
& \left\{\begin{array}{l|l}
\left(A^{0}(z), A_{\infty}(w)\right) \in \mathbb{O}_{V} \times \mathbb{O}_{V, \infty} & \begin{array}{l}
-A_{\infty, 2}+A^{0}(z) \text { is irreducible, } \\
A_{\infty, 1}=\operatorname{Res}_{z=\infty} A^{0}(z)
\end{array}
\end{array}\right\} / \mathrm{GL}(V),
\end{aligned}
$$

where $A_{\infty}(w)=A_{\infty, 1} w^{-1}+A_{\infty, 2} w^{-2}$. The symplectomorphism is given by

$$
S+\mathbb{O}_{V} \ni A(z) \mapsto\left(A(z)-S,-S w^{-2}+\left(\operatorname{Res}_{z=\infty} A(z)\right) w^{-1}\right) .
$$

The second component $-S w^{-2}+w^{-1} \operatorname{Res}_{z=\infty} A(z)$ on the right hand side is equal to the principal part of the Laurent expansion of $A(z) d z$ at $z \equiv w^{-1}=\infty$. Therefore we refer to $\mathfrak{M}^{\mathrm{irr}}\left(\mathbb{O}_{V}, \mathbb{O}_{V, \infty}\right)$ as the naive moduli space of irreducible systems having singularities on $D \cup\{\infty\}$ with truncated formal type $\left(\mathbb{O}_{V}, \mathbb{O}_{V, \infty}\right)$. Since the map

$$
\mathbb{O}_{V} \times \mathbb{O}_{V, \infty} \ni\left(A^{0}(z), A_{\infty}(w)\right) \mapsto A_{\infty, 1}-\operatorname{Res}_{z=\infty} A^{0}(z)
$$

is a $\mathrm{GL}(V)$-moment map, $\mathfrak{M}^{\mathrm{irr}}\left(\mathbb{O}_{V}, \mathbb{O}_{V, \infty}\right)$ is an open subset of the symplectic quotient of $\mathbb{O}_{V} \times \mathbb{O}_{V, \infty}$ by the GL( $\left.V\right)$-action, which was studied by Boalch [10].

Remark 5.12. If both $S$ and $T$ are semisimple, then we have $G_{S}=\prod_{s} \operatorname{GL}\left(V_{s}\right)$ and $G_{T}=\prod_{t} \mathrm{GL}\left(W_{t}\right)$. In such cases the open subset of

$$
\mu_{S}^{-1}\left(O_{S}\right) \cap \mu_{T}^{-1}\left(O_{T}\right) / G_{S} \times G_{T},
$$

given by all irreducible Harnad data with fixed $(V, W, S, T)$, is the Nakajima quiver variety [34] associated to some graph. Therefore Theorem 5.10 tells us that the two naive moduli spaces $\mathfrak{M}_{S}^{\text {irr }}\left(\mathbb{O}_{V}, O_{S}\right)$ and $\mathfrak{M}_{T}^{\text {irr }}\left(\mathbb{O}_{W}, O_{T}\right)$ are symplectomorphic to the same Nakajima quiver variety. It is a typical example of Boalch's 'different realizations' (see [13, §4.7] and also [12, Appendix A]). 
Remark 5.13. Assume $E=\{0\}$ and $\vec{l}=(1)$. Let $(V, W, 0, T, Q, P)$ be an irreducible Harnad datum with $S=0, V, W \neq 0$. Then $(V, A)=\Phi(V, W, 0, T, Q, P)$ and its Harnad dual $(W, B)=\mathrm{HD}(V, A)$ are written as

$$
A(z)=Q\left(z \operatorname{Id}_{W}-T\right)^{-1} P, \quad B(\zeta)=T+\frac{P Q}{\zeta} .
$$

Consider the operator

$$
\zeta\left(\frac{d}{d \zeta}-B(\zeta)+\zeta^{-1} \operatorname{Id}_{W}\right)=\zeta \frac{d}{d \zeta}-T \zeta-P Q+\operatorname{Id}_{W}
$$

corresponding to the system $B(\zeta)-\zeta^{-1} \mathrm{Id}_{W}$. The inverse Fourier-Laplace transform $\mathfrak{F}^{-1}: \zeta \mapsto-d / d z, d / d \zeta \mapsto z$ of it is given by

$$
\begin{aligned}
-\frac{d}{d z} z+T \frac{d}{d z}-P Q+\operatorname{Id}_{W} & =-\left(\operatorname{Id}_{W}+z \frac{d}{d z}\right)+T \frac{d}{d z}-P Q+\operatorname{Id}_{W} \\
& =-\left(z \operatorname{Id}_{W}-T\right) \frac{d}{d z}-P Q,
\end{aligned}
$$

which corresponds to the system $\widetilde{A}(z):=-\left(z \operatorname{Id}_{W}-T\right)^{-1} P Q$. The relation

$$
A(z) Q=Q\left(z \operatorname{Id}_{W}-T\right)^{-1} P Q=-Q \widetilde{A}(z)
$$

means that $Q: W \rightarrow V$ gives a morphism from $(W,-\tilde{A})$ to $(V, A)$. Note that $Q$ is surjective and $P$ is injective because the pairs $(\operatorname{Im} Q, W)$ and $(\operatorname{Ker} P, 0)$ are both subrepresentations of the irreducible Harnad datum $(V, W, 0, T, Q, P)$. In particular, $(V, A)$ is an irreducible quotient of $(W,-\tilde{A})$. So we have a commutative diagram

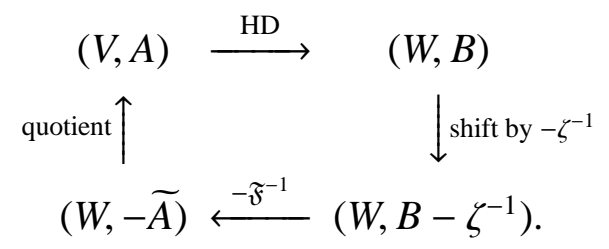

When $T$ is semisimple, a regular singular system of the form

$$
\left(z \operatorname{Id}_{W}-T\right) \frac{d u}{d z}=R u, \quad R \in \operatorname{End}(W)
$$

is called a system of Okubo normal form and has been studied by many researchers (see e.g. [8, 23, 36, 43]). Kawakami [27] further studied systems of the above form for arbitrary $T$ (then irregular singularities appear), and construct a functor from the category of triples $(W, T, R)$ to that of pairs $(V, A)$, which is defined as follows: for given triple $(W, T, R)$, write $R=P Q$, where $Q: W \rightarrow V:=W / \operatorname{Ker} R$ is the projection and $P: V \rightarrow W$ is the injection induced from $R$, and then send it to pair $\left(V, Q\left(z \operatorname{Id}_{W}-T\right)^{-1} P\right)$. Note that it coincides with the left vertical arrow in the previous diagram. 
Remark 5.14. So far we have restricted our Harnad data by Conditions (3.1) and (5.1) using some fixed $D, E, \vec{k}, \vec{l}$. Considering here various $D, E, \vec{k}, \vec{l}$ at once, we obtain the category $\mathcal{H}$ of all Harnad data, and similarly, the category $\mathcal{D}$ of pairs $(V, A)$ consisting of a finite-dimensional $\mathbb{C}$-vector space $V$ and an $\operatorname{End}(V)$-valued rational function $A(z)$ which is bounded locally near $\infty$. Then using Remark 4.9, (b), we can show that the functors $\Phi, \kappa, \mathrm{HD}$ are uniquely extended to functors $\Phi: \mathcal{H} \rightarrow \mathcal{D}, \kappa: \mathcal{D} \rightarrow$ $\mathcal{H}, \mathrm{HD}: \mathcal{D} \rightarrow \mathcal{D}$. All the results, except Theorem 5.10, obtained in this section can be rephrased in terms of these categories/functors.

\section{Generalized middle convolution}

For $\alpha \in \mathcal{E}_{\vec{l}}(\mathbb{C})$, we define the addition functor with $\alpha$ by

$$
a d d_{\alpha}: \vec{k}_{\vec{l}} \mathcal{D}_{\vec{k}} \mathcal{D}_{\vec{l}} ; \quad(W, B) \mapsto(W, B+\alpha) .
$$

Now the generalized middle convolution is defined as follows:

Definition 6.1. For $\alpha \in \mathcal{E}_{\vec{l}}(\mathbb{C})$, we define

$$
m c_{\alpha}:=\mathrm{HD} \circ \operatorname{add} d_{\alpha} \circ \mathrm{HD}:{ }_{\vec{l}} \mathcal{D}_{\vec{k}} \rightarrow{ }_{\vec{l}} \mathcal{D}_{\vec{k}},
$$

which we call the middle convolution functor with $\alpha$.

Example 6.2. (a) If $0 \in E, \alpha(\zeta)=\lambda / \zeta$ and $(V, A)$ is Fuchsian, $m c_{\alpha}(V, A)$ coincides with the original middle convolution $m c_{\lambda}(V, A)$.

(b) As a simple example, let us consider the case that $D, E=\{0\}$ and $(V, A)=$ $(\mathbb{C}, \alpha)$ is of rank 1. We omit the subscript $t=0$ as usual. By virtue of Example 5.3, (b), we already know that in this case the Harnad dual $(W, B)=\operatorname{HD}(\mathbb{C}, \alpha)$ is given by

$$
W=\mathbb{C}^{d}, \quad B(\zeta)=N+\frac{R}{\zeta},
$$

where $d$ is the pole order of $\alpha, N$ is the $d \times d$ nilpotent single Jordan block, and $R$ is defined by

$$
R=\left(\begin{array}{cccc}
0 & 0 & \cdots & 0 \\
\vdots & \vdots & & \vdots \\
0 & 0 & \cdots & 0 \\
\alpha_{d} & \alpha_{d-1} & \cdots & \alpha_{1}
\end{array}\right) \in \operatorname{End}\left(\mathbb{C}^{d}\right)
$$

Now let us compute the middle convolution $m c_{\lambda / \zeta}(\mathbb{C}, \alpha)=\operatorname{HD}\left(\mathbb{C}^{d}, B+\lambda / \zeta\right)$ with $\lambda / \zeta \neq 0$. If $\lambda+\alpha_{1} \neq 0$, the matrix $R+\lambda \operatorname{Id}_{\mathbb{C}^{d}}$ is invertible. Hence the canonical datum $\left(\mathbb{C}^{d}, V^{\prime}, 0, P^{\prime}, Q^{\prime}\right)$ for $\zeta^{-1}\left(R+\lambda \operatorname{Id}_{\mathbb{C}^{d}}\right)$ is given by

$$
V^{\prime}=\mathbb{C}^{d}, \quad P^{\prime}=R+\lambda \mathrm{Id}_{\mathbb{C}^{d}}, \quad Q^{\prime}=\mathrm{Id}_{\mathbb{C}^{d}}
$$


whence

$$
m c_{\lambda / \zeta}(\mathbb{C}, \alpha)=\left(\mathbb{C}^{d}, Q^{\prime}\left(z \operatorname{Id}_{\mathbb{C}^{d}}-N\right)^{-1} P^{\prime}\right)=\left(\mathbb{C}^{d},\left(z \operatorname{Id}_{\mathbb{C}^{d}}-N\right)^{-1}\left(R+\lambda \operatorname{Id}_{\mathbb{C}^{d}}\right)\right)
$$

As a matrix, $\left(z \operatorname{Id}_{\mathbb{C}^{d}}-N\right)^{-1}\left(R+\lambda \operatorname{Id}_{\mathbb{C}^{d}}\right)$ has the $(i, j)$-entry given by

$$
\left[\left(z \operatorname{Id}_{\mathbb{C}^{d}}-N\right)^{-1}\left(R+\lambda \operatorname{Id}_{\mathbb{C}^{d}}\right)\right]_{i, j}= \begin{cases}\frac{\alpha_{d-j+1}}{z^{d-i+1}}+\frac{\lambda}{z^{j-i+1}} & i \leq j \\ \frac{\alpha_{d-j+1}}{z^{d-i+1}} & i>j .\end{cases}
$$

If $\lambda+\alpha_{1}=0$, the subspace $\operatorname{Ker}\left(R+\lambda \operatorname{Id}_{\mathbb{C}^{d}}\right)$ is generated by the $d$-th coordinate vector in $\mathbb{C}^{d}$ and hence the projection $\mathbb{C}^{d} \rightarrow \mathbb{C}^{d-1}$ killing the $d$-th component gives an isomorphism $V^{\prime}=\mathbb{C}^{d} / \operatorname{Ker}\left(R+\lambda \operatorname{Id}_{\mathbb{C}^{d}}\right) \simeq \mathbb{C}^{d-1}$. Under this identification, the decomposition

$$
R+\lambda \operatorname{Id}_{\mathbb{C}^{d}}=\left(\begin{array}{ccc}
\lambda & & 0 \\
& \ddots & \\
0 & & \lambda \\
\alpha_{d} & \cdots & \alpha_{2}
\end{array}\right)\left(\begin{array}{cccc}
1 & 0 & \cdots & 0 \\
& \ddots & \ddots & \vdots \\
0 & & 1 & 0
\end{array}\right)
$$

gives the matrices $P^{\prime}$ and $Q^{\prime}$. The middle convolution is given by

$$
m c_{\lambda / \zeta}(\mathbb{C}, \alpha)=\left(\mathbb{C}^{d-1}, Q^{\prime}\left(z \operatorname{Id}_{\mathbb{C}^{d}}-N\right)^{-1} P^{\prime}\right)
$$

As in Remark 5.13, we have

$$
\left[Q^{\prime}\left(z \operatorname{Id}_{\mathbb{C}^{d}}-N\right)^{-1} P^{\prime}\right] Q^{\prime}=Q^{\prime}\left[\left(z \operatorname{Id}_{\mathbb{C}^{d}}-N\right)^{-1} P^{\prime} Q^{\prime}\right]
$$

Since $Q^{\prime}=\left(\operatorname{Id}_{\mathbb{C}^{d-1}} 0\right)$, we see that the $(i, j)$-entry of $Q^{\prime}\left(z \operatorname{Id}_{\mathbb{C}^{d}}-N\right)^{-1} P^{\prime}$ is the same as that of $\left(z \operatorname{Id}_{\mathbb{C}^{d}}-N\right)^{-1} P^{\prime} Q^{\prime}$, which is given by 6.1 .

We give three basic properties of the middle convolution. First rephrasing Theorem 5.8, we have the following:

Corollary 6.3. If $(V, A) \in \in_{\vec{l}} \mathcal{D}_{\vec{k}}$ is irreducible and $(V, A) \nsim(\mathbb{C}, s)$ for any $s \in E$, then $m c_{0}(V, A) \sim(V, A)$.

Furthermore, Theorem 5.8 also implies the following:

Corollary 6.4. Suppose that a pair $(V, A) \in{ }_{\vec{l}} \mathcal{D}_{\vec{k}}$ and $\alpha \in \mathcal{E}_{\vec{l}}(\mathbb{C})$ satisfy the following conditions:

(a) $(V, A)$ is irreducible;

(b) $(V, A) \times(\mathbb{C}, s)$ for any $s \in E$; 
(c) $m c_{\alpha}(V, A) \neq(0,0)$.

Then $m c_{\alpha}(V, A)$ is also irreducible and

$$
m c_{\beta} \circ m c_{\alpha}(V, A) \sim m c_{\alpha+\beta}(V, A)
$$

for any $\beta \in \mathcal{E}_{\vec{l}}(\mathbb{C})$.

Proof. Clearly the addition functor $a d d_{\alpha}$ preserves the irreducibility. Therefore Theorem 5.8 implies that $a d d_{\alpha} \circ \operatorname{HD}(V, A)$ is irreducible, and further that $m c_{\alpha}(V, A)$ is irreducible if $a d d_{\alpha} \circ \operatorname{HD}(V, A) \nsim(\mathbb{C}, t)$ for any $t \in D$, or equivalently (by Corollary 5.9), if $m c_{\alpha}(V, A) \neq(0,0)$. Furthermore, under the same assumption we have

$$
\begin{aligned}
m c_{\beta} \circ m c_{\alpha}(V, A) & =\mathrm{HD} \circ a d d_{\beta} \circ(\mathrm{HD} \circ \mathrm{HD}) \circ a d d_{\alpha} \circ \mathrm{HD}(V, A) \\
& \sim \mathrm{HD} \circ a d d_{\beta} \circ a d d_{\alpha} \circ \operatorname{HD}(V, A) \\
& =\mathrm{HD} \circ a d d_{\alpha+\beta} \circ \operatorname{HD}(V, A)=m c_{\alpha+\beta}(V, A) .
\end{aligned}
$$

Theorem 5.10 and Remark 5.11 imply the following (the proof is immediate):

Corollary 6.5. Suppose that a pair $(V, A) \in{ }_{\vec{l}} \mathcal{D}_{\vec{k}}$ and $\alpha \in \mathcal{E}_{\vec{l}}(\mathbb{C})$ satisfy all the assumptions in Corollary 6.4 Let $\mathbb{O} \times \mathbb{O}_{\infty} \subset \mathcal{E}_{\vec{k}}(V) \times \mathfrak{g}_{2}^{*}(V)$ be the truncated formal type of $A(z)$, namely, the $G_{\vec{k}}(V) \times G_{2}(V)$-coadjoint orbit such that $A(z)$ gives a point in $\mathfrak{M}^{\mathrm{irr}}\left(\mathbb{O}, \mathbb{O}_{\infty}\right)$. Similarly, let $\left(V^{\alpha}, A^{\alpha}\right):=m c_{\alpha}(V, A)$ and let $\mathbb{O}^{\alpha} \times \mathbb{O}_{\infty}^{\alpha} \subset \mathcal{E}_{\vec{k}}\left(V^{\alpha}\right) \times \mathfrak{g}_{2}^{*}\left(V^{\alpha}\right)$ be the truncated formal type of $A^{\alpha}(z)$. Then the middle convolution induces a symplectomorphism

$$
m c_{\alpha}: \mathfrak{M}^{\mathrm{irr}}\left(\mathbb{O}, \mathbb{O}_{\infty}\right) \stackrel{\simeq}{\rightarrow} \mathfrak{M}^{\mathrm{irr}}\left(\mathbb{O}^{\alpha}, \mathbb{O}_{\infty}^{\alpha}\right)
$$

The rest of this section is devoted to study the behavior of truncated formal type $\left(\mathbb{O}, \mathbb{O}_{\infty}\right) \mapsto\left(\mathbb{O}^{\alpha}, \mathbb{O}_{\infty}^{\alpha}\right)$ under the middle convolution $m c_{\alpha}$. The following example treats the behavior of $\mathbb{O}_{\infty}$ in some special, but important case.

Example 6.6. Suppose that $\mathbb{O}_{\infty}=w^{-1} \lambda \operatorname{Id}_{V}$ for some $\lambda \neq 0$ (where $w=z^{-1}$ is the indeterminate for $\mathbb{O}_{\infty}$ as in Remark 5.11). Then for any representative $A(z)$ of a point in $\mathfrak{M}^{\mathrm{irr}}\left(\mathbb{O}, \mathbb{O}_{\infty}\right)$, we have

$$
S=0, \quad \operatorname{Res}_{z=\infty} A(z)=\lambda \operatorname{Id}_{V}
$$

Let $(V, W, T, Q, P)$ be the canonical datum for $A(z)$. Since $Q P=-\lambda \operatorname{Id}_{V}$ and $\lambda \neq 0$, we see that $Q$ is surjective and $P$ is injective. Set $V^{\prime}=\operatorname{Coker} P$ and let $Q^{\prime}: W \rightarrow V^{\prime}$ be the natural projection. Then we have an exact sequence

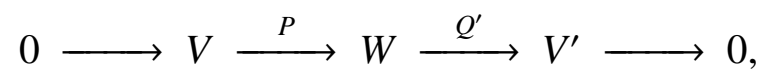


and $-\lambda^{-1} Q$ gives a splitting of it. Let $P^{\prime}: V^{\prime} \rightarrow W$ be the injection such that $\lambda^{-1} P^{\prime}$ is the homomorphism associated to this splitting;

$$
P\left(-\lambda^{-1} Q\right)+\lambda^{-1} P^{\prime} Q^{\prime}=\operatorname{Id}_{W}
$$

namely,

$$
P^{\prime} Q^{\prime}-P Q=\lambda \operatorname{Id}_{W}
$$

Then one can easily see that $\operatorname{Im} P=\operatorname{Ker}\left(P Q+\lambda \operatorname{Id}_{W}\right)$ and $Q^{\prime}$ coincides with the homomorphism induced from $P Q+\lambda \mathrm{Id}_{W}$. This observation shows that the datum $\left(V^{\prime}, W, T, Q^{\prime}, P^{\prime}\right)$ coincides with the canonical datum for $m c_{\lambda / \zeta}(V, A)$. By the definition we have $Q^{\prime} P^{\prime}=\lambda \operatorname{Id}_{V^{\prime}}$, and hence $\mathbb{O}_{\infty}^{\lambda / \zeta}=-w^{-1} \lambda \operatorname{Id}_{V^{\lambda / \zeta}}$. Thus we see that the middle convolution $m c_{\lambda / \zeta}$ induces a symplectomorphism

$$
\mathfrak{M}^{\mathrm{irr}}\left(\mathbb{O}, w^{-1} \lambda \operatorname{Id}_{V}\right) \stackrel{\simeq}{\rightarrow} \mathfrak{M}^{\mathrm{irr}}\left(\mathbb{O}^{\lambda / \zeta},-w^{-1} \lambda \operatorname{Id}_{V^{\lambda / \zeta}}\right),
$$

and $\operatorname{dim} V^{\lambda / \zeta}=\operatorname{dim} W-\operatorname{dim} V$. We will use it in the next section.

From now on, we mainly treat systems satisfying the following property:

Definition 6.7. An element in $\mathcal{E}_{k}(V)$ of the form

$$
\Lambda(z)=\bigoplus_{\lambda \in \Sigma}\left(\lambda(z) \operatorname{Id}_{V_{\lambda}}+\frac{\Gamma_{\lambda}}{z}\right),
$$

associated to

- a finite subset $\Sigma \subset \mathcal{E}_{k}(\mathbb{C})$ whose elements are all residue-free;

- a decomposition $V=\bigoplus_{\lambda \in \Sigma} V_{\lambda}$ by nonzero subspaces $V_{\lambda}, \lambda \in \Sigma$; and

- matrices $\Gamma_{\lambda} \in \operatorname{End}\left(V_{\lambda}\right), \lambda \in \Sigma$,

is called a Hukuhara-Turrittin-Levelt normal form, or simply, a normal form.

An element $A(z) \in \mathcal{E}_{k}(V)$ is said to have a normal form if it is equivalent to some normal form under the $G_{k}(V)$-action. A normal form $\Lambda(z)$ equivalent to $A(z)$ (which is unique up to $\mathrm{GL}(V)$-conjugation by Proposition 6.8 below) is called the normal form of $A(z)$, and each $\lambda \in \Sigma$ is called a spectrum of $A(z)$.

A system $A(z) \in \mathcal{E}_{\vec{k}}(V)$, or a pair $(V, A) \in{ }_{\vec{l}} \mathcal{D}_{\vec{k}}$, is said to have a normal form at $t \in D$ if its $\mathcal{E}_{k_{t}}(V)$-component $A_{t}(z)$ has a normal form.

Note that for any normal form $\Lambda(z)$, its coefficient matrices

$$
\Lambda_{i} \in \operatorname{End}(V), \quad \Lambda(z)=\sum_{i=1}^{k} \Lambda_{i} z^{-i}
$$

satisfy the following properties: 
(a) $\Lambda_{1}, \ldots, \Lambda_{k}$ commute with one another;

(b) $\Lambda_{2}, \ldots, \Lambda_{k}$ are all semisimple.

Each simultaneous eigenvalues $\left(\lambda_{2}, \ldots, \lambda_{k}\right)$ of $\left(\Lambda_{2}, \ldots, \Lambda_{k}\right)$ give a spectrum of $\Lambda(z)$ by $\lambda(z)=\sum_{i=2}^{k} \lambda_{i} z^{-i}$, and the subspace $V_{\lambda}$ is given by the corresponding simultaneous eigenspace.

Proposition 6.8. If two normal forms $\Lambda(z), \Lambda^{\prime}(z)$ are contained in the same $G_{k}(V)$ coadjoint orbit, then there exists $a \in \mathrm{GL}(V)$ such that $\Lambda^{\prime}(z)=a \Lambda(z) a^{-1}$.

Proof. Let $\Lambda(z)=\sum \Lambda_{i} z^{-i}, \Lambda^{\prime}(z)=\sum \Lambda_{i}^{\prime} z^{-i}$ and suppose that there exists $g(z)=$ $\sum_{i} g_{i} z^{i} \in G_{k}(V)$ such that $g \cdot \Lambda=\Lambda^{\prime}$. It means $g(z) \Lambda(z) z^{k}=\Lambda^{\prime}(z) g(z) z^{k}$ modulo $z^{k}$, which can be written as

$$
\sum_{i=0}^{k-l}\left(\Lambda_{l+i} g_{i}-g_{i} \Lambda_{l+i}^{\prime}\right)=0, \quad l=1,2, \ldots, k .
$$

Looking at the relation for $l=k$, we obtain $g_{0} \Lambda_{k}=\Lambda^{\prime} g_{0}$. So taking the conjugation by $g_{0}$ on both sides of the relation $g \cdot \Lambda=\Lambda^{\prime}$, we may assume that $g_{0}=1$ and $\Lambda_{k}=\Lambda_{k}^{\prime}$. Set $\mathfrak{h}_{k+1}:=\mathfrak{g l}(V)$ and inductively

$$
\mathfrak{h}_{l}:=\operatorname{Ker}\left(\left.\operatorname{ad}_{\Lambda_{l}}\right|_{\mathfrak{h}_{l+1}}\right), \quad \mathfrak{h}_{l}^{\prime}:=\operatorname{Im}\left(\left.\operatorname{ad}_{\Lambda_{l}}\right|_{\mathfrak{h}_{l+1}}\right), \quad l=1,2, \ldots, k
$$

Note that $\mathfrak{h}_{l}^{\prime} \subset \mathfrak{h}_{l+1}$ for $l \geq 1$ as $\Lambda_{1}, \ldots, \Lambda_{k}$ commute with one another. Moreover, since $\Lambda_{2}, \ldots, \Lambda_{k}$ are all semisimple, we have

$$
\mathfrak{h}_{l+1}=\mathfrak{h}_{l} \oplus \mathfrak{h}_{l}^{\prime}, \quad l=2, \ldots, k
$$

Now looking at the relation for $l=k-1$, we have

$$
\left[\Lambda_{k}, g_{1}\right] \in \mathfrak{h}_{k}^{\prime}, \quad \Lambda_{k-1}, \Lambda_{k-1}^{\prime} \in \mathfrak{h}_{k}, \quad\left[\Lambda_{k}, g_{1}\right]+\Lambda_{k-1}=\Lambda_{k-1}^{\prime}
$$

Since $\mathfrak{g l}(V)=\mathfrak{h}_{k}^{\prime} \oplus \mathfrak{h}_{k}$, we obtain

$$
\left[\Lambda_{k}, g_{1}\right]=0, \quad \Lambda_{k-1}=\Lambda_{k-1}^{\prime}
$$

The first relation means $g_{1} \in \mathfrak{h}_{k}$. Next looking at the relation for $l=k-2$, we obtain

$$
\left[\Lambda_{k}, g_{2}\right] \in \mathfrak{h}_{k}^{\prime}, \quad\left[\Lambda_{k-1}, g_{1}\right] \in \mathfrak{h}_{k-1}^{\prime}, \quad\left[\Lambda_{k}, g_{2}\right]+\left[\Lambda_{k-1}, g_{1}\right]+\Lambda_{k-2}=\Lambda_{k-2}^{\prime}
$$

Since $\mathfrak{g l}(V)=\mathfrak{h}_{k}^{\prime} \oplus \mathfrak{h}_{k-1}^{\prime} \oplus \mathfrak{h}_{k-1}$ and $\Lambda_{k-2}, \Lambda_{k-2}^{\prime} \in \mathfrak{h}_{k-1}$, we obtain

$$
\left[\Lambda_{k}, g_{2}\right]=0, \quad\left[\Lambda_{k-1}, g_{1}\right]=0, \quad \Lambda_{k-2}=\Lambda_{k-2}^{\prime}
$$


The first two relations mean $g_{2} \in \mathfrak{h}_{k}, g_{1} \in \mathfrak{h}_{k-1}$.

Iterating the argument inductively, we finally obtain

$$
g_{i} \in \mathfrak{h}_{i+1}, \quad \Lambda_{i}=\Lambda_{i}^{\prime}, \quad i=1, \ldots, k-1
$$

from the decomposition $\mathfrak{g l}(V)=\mathfrak{h}_{k}^{\prime} \oplus \mathfrak{h}_{k-1}^{\prime} \oplus \cdots \oplus \mathfrak{h}_{2}^{\prime} \oplus \mathfrak{h}_{2}$.

Remark 6.9. If the leading term of $A(z)=\sum_{i} A_{i} z^{-i} \in \mathcal{E}_{k}(V)$ is diagonalizable with distinct eigenvalues, then $A(z)$ has a normal form $\Lambda(z)=\sum_{i} \Lambda_{i} z^{-i}$, where $\operatorname{ord}(\Lambda)=$ $\operatorname{ord}(A)(=: d)$ and $\Lambda_{i}$ is given by the $\operatorname{Ker}\left(\operatorname{ad}_{A_{d}}\right)$-part of $A_{i}$ relative to the decomposition $\mathfrak{g l}(V)=\operatorname{Ker}\left(\operatorname{ad}_{A_{d}}\right) \oplus \operatorname{Im}\left(\operatorname{ad}_{A_{d}}\right)$ (i.e., the 'diagonal part' of $\left.A_{i}\right)$.

Conversely, one can easily show that if $A(z) \in \mathcal{E}_{k}(V)$ has a normal form, then its leading term is semisimple.

Remark 6.10. Of course we can also define a similar notion for the singularity at $\infty$; $(V, A) \in{ }_{\vec{l}} \mathcal{D}_{\vec{k}}$ is said to have a normal form at $\infty$ if the principal part

$$
A_{\infty}(w)=-S w^{-2}+w^{-1} \operatorname{Res}_{z=\infty} A(z) \in \mathcal{E}_{2}(V)
$$

of the Laurent expansion of $A(z) d z$ at $\infty$ has a normal form. Here one can observe that in general, an element $S w^{-2}+R w^{-1} \in \mathcal{E}_{2}(V)$ has a normal form if and only if $S$ is semisimple. Hence $(V, A) \in_{\vec{l}} \mathcal{D}_{\vec{k}}$ has a normal form at $\infty$ if and only if $S=\lim _{z \rightarrow \infty} A(z)$ is semisimple, which is equivalent to that $\operatorname{HD}(V, A)$ is Fuchsian at any $s \in E$ if $(V, A)$ is irreducible.

Remark 6.11. Let $\mathcal{F}:=\mathbb{C}((z))$ be the field of formal Laurent series and consider each $A(z) \in \mathcal{E}_{k}(V)$ as an element of $\operatorname{End}_{\mathcal{F}}(V \otimes \mathcal{F}) \simeq \mathfrak{g l}(V) \otimes \mathcal{F}$, on which the group $\operatorname{Aut}_{\mathcal{F}}(V \otimes \mathcal{F})$ acts as gauge transformations;

$$
A \mapsto g[A]:=g A g^{-1}+\frac{d g}{d z} g^{-1}, \quad g \in \operatorname{Aut}_{\mathcal{F}}(V \otimes \mathcal{F}) .
$$

A fundamental fact in the Hukuhara-Turrittin-Levelt theory of meromorphic linear ordinary differential equations $[24,31,39]$ is that for any system $A(z) \in \mathfrak{g l}(V) \otimes \mathcal{F}$, there exists a positive integer $b$ such that the pull back of the form $A(z) d z$ via the ramified covering map $f_{b}: z \mapsto z^{b}$ is equivalent to some normal form $\Lambda(z) d z$ under the $\operatorname{Aut}_{\mathcal{F}}(V \otimes \mathcal{F})$-action. For this reason, a system $A(z)$ is said to be unramified if it is equivalent to some normal form $\Lambda(z)$ under the $\operatorname{Aut}_{\mathcal{F}}(V \otimes \mathcal{F})$-action (i.e., in the case of $b=1$ ).

It is also known (see [6, §6-7]) that two normal forms $\Lambda(z)=\sum \Lambda_{i} z^{-i}, \Lambda^{\prime}(z)=$ $\sum \Lambda_{i}^{\prime} z^{-i}$ are equivalent under the $\operatorname{Aut}_{\mathcal{F}}(V \otimes \mathcal{F})$-action if and only if those have the same pole order and there exists $a \in \mathrm{GL}(V)$ such that

$$
a \Lambda_{i} a^{-1}=\Lambda_{i}^{\prime}, i \geq 2, \quad a \exp \left(2 \pi \sqrt{-1} \Lambda_{1}\right) a^{-1}=\exp \left(2 \pi \sqrt{-1} \Lambda_{1}^{\prime}\right) .
$$


It implies that for any unramified system $A(z) \in \mathfrak{g l}(V) \otimes \mathcal{F}$, there exists a unique normal form $\Lambda(z)$ equivalent to $A(z)$ such that the real parts of all the eigenvalues of the residue $\Lambda_{1}=\operatorname{Res}_{z=0} \Lambda(z)$ are in [0,1). A normal form satisfying such a condition is said to be reduced.

Note that in the above we consider formal meromorphic gauge transformations. Replacing the action of $\operatorname{Aut}_{\mathcal{F}}(V \otimes \mathcal{F})$ with that of the subgroup $\operatorname{Aut}_{\mathbb{C}[z]]}(V \otimes \mathbb{C}[[z]])$ (in other words, considering only formal gauge transformations) in the above definition of unramifiedness, we obtain a notion close to the one in Definition 6.7; in fact, the following two conditions for a system $A(z) \in \mathcal{E}_{k}(V)$ are equivalent:

(a) $A(z)$ is equivalent to a reduced normal form under the $G_{k}(V)$-action;

(b) $A(z)$ is equivalent to a reduced normal form under the $\operatorname{Aut}_{\mathbb{C}[z]]}(V \otimes \mathbb{C}[[z]])$ action.

The direction (b) $\Rightarrow$ (a) is clear because the relation $g[A]=\Lambda$ induces the relation $\bar{g} \cdot A=\Lambda$ in $\mathcal{E}_{k}(V)$, where $\bar{g}(z) \in G_{k}(V)$ is the element induced from $g$ by taking modulo $z^{k}$ (so this direction is also true in the non-reduced case). The proof of the direction (a) $\Rightarrow(\mathrm{b})$ is as follows. For given relation $g \cdot A=\Lambda$ in $\mathcal{E}_{k}(V)$, consider $g$ as an element of $\operatorname{Aut}_{\mathbb{C}[[z]]}(V \otimes \mathbb{C}[[z]])$. Then it is easy to see that $g[A]-\Lambda \in \mathfrak{g l}(V) \otimes \mathbb{C}[[z]]$. Now use [7, Lemma 1.4.1].

Note that Condition (b) is not equivalent to the unramifiedness. For instance, let $\Gamma$ be the $2 \times 2$ nilpotent single Jordan block and set $\Lambda(z):=\Gamma z^{-1}$ which is a normal form by definition. Take $g(z):=\operatorname{diag}\left(1, z^{k}\right)$ for some integer $k>1$. Then the unramified element $A(z):=g[\Lambda]$ has pole order $k$ and no $\mathbb{C}[[z]]$-part, so it is contained in $\mathcal{E}_{k}\left(\mathbb{C}^{2}\right)$. However it is not equivalent to a normal form under the $\operatorname{Aut}_{\mathbb{C}[[z]]}(V \otimes \mathbb{C}[[z]])$-action because its leading term is nilpotent.

Recall that by Theorem 4.14, each $G_{\vec{k}}(V)$-coadjoint orbit can be described as the symplectic quotient $\mu_{T}^{-1}(O)^{T \text {-st }} / G_{T}$ for some vector space $W$, an endomorphism $T \in \operatorname{End}(W)$ and a $G_{T}$-coadjoint orbit $O \subset \mathfrak{g}_{T}^{*}$ via the map $\Phi_{T}$. We denote it by $\mu_{T}^{-1}(O)_{V}^{T-\text { st }} / G_{T}$ when we want to emphasize the vector space $V$. In the two lemmas below, we assume for simplicity that $D=\{0\}$ and omit the subscript $t=0$ as usual. Also for $T \in \mathfrak{g l}(W)$, we denote by $p_{T}: \mathfrak{g l}(W) \rightarrow \mathfrak{g}_{T}^{*}$ the natural projection onto the dual of the centralizer $\mathfrak{g}_{T}$ of $T$ as before.

Lemma 6.12. Let $W$ be a nonzero finite-dimensional $\mathbb{C}$-vector space, $N \in \operatorname{End}(W)$ be a nilpotent endomorphism with $N^{k}=0$, and $O \subset \mathfrak{g}_{N}^{*}$ be a $G_{N^{-}}$coadjoint orbit. Suppose that finite-dimensional $\mathbb{C}$-vector spaces $V, V^{\prime} \neq 0$ and $\alpha \in \mathbb{C}$ satisfy the following conditions: 
(a) $\Phi_{N}\left(\mu_{N}^{-1}(O)_{V}^{N \text {-st }} / G_{N}\right)$ contains a normal form;

(b) $\mu_{N}^{-1}\left(O+p_{N}\left(\alpha \operatorname{Id}_{W}\right)\right)_{V^{\prime}}^{N \text {-st }}$ is non-empty,

Then the orbit $\Phi_{N}\left(\mu_{N}^{-1}\left(O+p_{N}\left(\alpha \operatorname{Id}_{W}\right)\right)_{V^{\prime}}^{N \text {-st }} / G_{N}\right)$ contains a normal form which has the same nonzero spectra as that in (a).

Proof. By the assumption, the image of $\mu_{N}^{-1}(O)_{V}^{N \text {-st }} / G_{N}$ under the map $\Phi_{N}$ contains a normal form

$$
\Lambda(z)=\bigoplus_{\lambda \in \Sigma}\left(\lambda(z) \operatorname{Id}_{V_{\lambda}}+\frac{\Gamma_{\lambda}}{z}\right), \quad \lambda(z)=\sum_{i=2}^{k} \lambda_{i} z^{-i}, V=\bigoplus_{\lambda \in \Sigma} V_{\lambda}, \Gamma_{\lambda} \in \operatorname{End}\left(V_{\lambda}\right) .
$$

In what follows we assume $0 \in \Sigma$. Replacing $\Sigma$ with $\Sigma \cup\{0\}$ and setting $V_{0}:=0, \Gamma_{0}:=$ 0 make the argument below work well also in the case $0 \notin \Sigma$.

Set $d_{\lambda}:=\operatorname{ord}\left(\lambda+\Gamma_{\lambda} / z\right)$. By Remark 4.9, we see that the canonical datum for $\Lambda(z)$ is given by the direct sum of those $\left(V_{\lambda}, W_{\lambda}, N_{\lambda}, Q_{\lambda}, P_{\lambda}\right)$ for $\lambda(z) \operatorname{Id}_{V_{\lambda}}+\Gamma_{\lambda} / z$. The direct summand for $\lambda \neq 0$ is given by

$$
\begin{aligned}
& W_{\lambda}=V_{\lambda} \otimes \mathbb{C}^{d_{\lambda}}, \\
& Q_{\lambda}=\left(\begin{array}{lllll}
\lambda_{d_{\lambda}} \operatorname{Id}_{V_{\lambda}} & \lambda_{d_{\lambda}-1} \operatorname{Id}_{V_{\lambda}} & \cdots & \lambda_{2} \operatorname{Id}_{V_{\lambda}} & \Gamma_{\lambda}
\end{array}\right) \in \operatorname{Hom}\left(W_{\lambda}, V_{\lambda}\right), \\
& P_{\lambda}=\left(\begin{array}{c}
0 \\
\vdots \\
0 \\
\operatorname{Id}_{V_{\lambda}}
\end{array}\right) \in \operatorname{Hom}\left(V_{\lambda}, W_{\lambda}\right), \quad N_{\lambda}=\left(\begin{array}{cccc}
0 & \operatorname{Id}_{V_{\lambda}} & & 0 \\
& 0 & \ddots & \\
& & \ddots & \operatorname{Id}_{V_{\lambda}} \\
0 & & & 0
\end{array}\right) \in \operatorname{End}\left(W_{\lambda}\right) \text {, }
\end{aligned}
$$

and for $\lambda=0$, given by

$$
\begin{gathered}
W_{0}=V_{0} / \operatorname{Ker} \Gamma_{0}, \quad N_{0}=0, \\
Q_{0}: W_{0} \stackrel{\Gamma_{0}}{\longrightarrow} V_{0}, \quad P_{0}: V_{0} \stackrel{\text { projection }}{\longrightarrow} W_{0} .
\end{gathered}
$$

By Proposition 4.1, we may assume that $W=\bigoplus_{\lambda} W_{\lambda}$ and $N=\bigoplus_{\lambda} N_{\lambda}$. We set $P:=\bigoplus_{\lambda} P_{\lambda}, Q:=\bigoplus_{\lambda} Q_{\lambda}$.

Now take a point $\left(Q^{\prime}, P^{\prime}\right) \in \mu_{N}^{-1}\left(O+p_{N}\left(\alpha \operatorname{Id}_{W}\right)\right)_{V^{\prime}}^{N \text {-st }}$. By using the $G_{N^{-}}$-action, we may assume that $\mu_{N}\left(Q^{\prime}, P^{\prime}\right)=\mu_{N}(Q, P)+p_{N}\left(\alpha \operatorname{Id}_{W}\right)$, i.e.,

$$
P^{\prime} Q^{\prime}=P Q-\alpha \operatorname{Id}_{W}+[N, X]
$$

for some $X \in \operatorname{End}(W)$. Let us write it as

$$
\begin{aligned}
& \left(P^{\prime} Q^{\prime}\right)_{\lambda \lambda}=P_{\lambda} Q_{\lambda}-\alpha \operatorname{Id}_{W_{\lambda}}+\left[N_{\lambda}, X_{\lambda \lambda}\right], \\
& \left(P^{\prime} Q^{\prime}\right)_{\lambda \mu}=N_{\lambda} X_{\lambda \mu}-X_{\lambda \mu} N_{\mu}, \quad \lambda \neq \mu,
\end{aligned}
$$


where the subscript ' $\lambda \mu$ ' means the $\operatorname{Hom}\left(W_{\mu}, W_{\lambda}\right)$-block component. By Lemma 3.7 , the restriction $\left.Q^{\prime}\right|_{\operatorname{Ker} N}$ is injective. Regarding $\operatorname{Ker} N_{\lambda}$ as a subspace of $\operatorname{Ker} N \subset W$ using the direct sum decomposition, we set $V_{\lambda}^{\prime}:=Q^{\prime}\left(\operatorname{Ker} N_{\lambda}\right)$ for $\lambda \neq 0$ and

$$
V_{0}^{\prime}:=V^{\prime} / \bigoplus_{\lambda \neq 0} V_{\lambda}^{\prime}
$$

Since the composite of $\left.P^{\prime} Q^{\prime}\right|_{\operatorname{Ker} N_{\lambda}}: \operatorname{Ker} N_{\lambda} \rightarrow W$ and the projection $\pi_{0}: W \rightarrow W_{0}$ is

$$
\left.\left(P^{\prime} Q^{\prime}\right)_{0 \lambda}\right|_{\operatorname{Ker} N_{\lambda}}=\left.\left(N_{0} X_{0 \lambda}-X_{0 \lambda} N_{\lambda}\right)\right|_{\operatorname{Ker} N_{\lambda}}=0
$$

for $\lambda \neq 0$, the map $\pi_{0} \circ P^{\prime}: V^{\prime} \rightarrow W_{0}$ descends to a map

$$
V_{0}^{\prime}=V^{\prime} / \bigoplus_{\lambda \neq 0} V_{\lambda}^{\prime} \rightarrow W_{0}
$$

which we denote by $P_{0}^{\alpha}$. Note that $\operatorname{Ker} \pi_{0} \supset \operatorname{Im} N$ since $N_{0}=0$. Hence by Lemma 3.7, $\pi_{0} \circ P^{\prime}$ is surjective and hence so is $P_{0}^{\alpha}$. Also we define $Q_{0}^{\alpha}: W_{0} \rightarrow V_{0}^{\prime}$ by the composite

$$
W_{0} \stackrel{\text { inclusion }}{\longrightarrow} W \stackrel{Q^{\prime}}{\rightarrow} V^{\prime} \stackrel{\text { projection }}{\longrightarrow} V_{0}^{\prime},
$$

which is injective since $W_{0} \subset \operatorname{Ker} N$. Clearly the pair $\left(Q_{0}^{\alpha}, P_{0}^{\alpha}\right)$ satisfies

$$
P_{0}^{\alpha} Q_{0}^{\alpha}=\left(P^{\prime} Q^{\prime}\right)_{00}=P_{0} Q_{0}-\alpha \operatorname{Id}_{W_{0}} .
$$

For $\lambda \neq 0$, let $\left(V_{\lambda}, W_{\lambda}^{\alpha}, N_{\lambda}^{\alpha}, Q_{\lambda}^{\alpha}, P_{\lambda}^{\alpha}\right)$ be the canonical datum for the system

$$
\lambda(z) \operatorname{Id}_{V_{\lambda}}+\frac{\Gamma_{\lambda}}{z}-\frac{d_{\lambda} \alpha}{z} \operatorname{Id}_{V_{\lambda}} \in \mathcal{E}_{k}\left(V^{\lambda}\right) .
$$

By Remark 4.9 we have $\left(W_{\lambda}^{\alpha}, N_{\lambda}^{\alpha}\right)=\left(W_{\lambda}, N_{\lambda}\right)$ and

$$
Q_{\lambda}^{\alpha}=Q_{\lambda}-\left(\begin{array}{llll}
0 & \cdots & 0 & d_{\lambda} \alpha \operatorname{Id}_{V_{\lambda}}
\end{array}\right), \quad P_{\lambda}^{\alpha}=P_{\lambda} .
$$

Now identifying $V_{\lambda}^{\prime}$ with $V_{\lambda}$ by using the injection $\left.Q^{\prime}\right|_{\text {Ker } N_{\lambda}}$ for $\lambda \neq 0$, set

$$
\left(V^{\prime}, W, N, Q^{\alpha}, P^{\alpha}\right):=\left(V_{0}^{\prime}, W_{0}, 0, Q_{0}^{\alpha}, P_{0}^{\alpha}\right) \oplus \bigoplus_{\lambda \neq 0}\left(V_{\lambda}, W_{\lambda}, N_{\lambda}, Q_{\lambda}^{\alpha}, P_{\lambda}^{\alpha}\right) .
$$

Then it is stable by Remark 4.9 and satisfies

$$
\Phi_{N}\left(Q^{\alpha}, P^{\alpha}\right)=\frac{Q_{0}^{\alpha} P_{0}^{\alpha}}{z} \oplus \bigoplus_{\lambda \neq 0}\left(\lambda(z) \operatorname{Id}_{V_{\lambda}}+\frac{\Gamma_{\lambda}}{z}-\frac{d_{\lambda} \alpha}{z} \operatorname{Id}_{V_{\lambda}}\right) \in \mathcal{E}_{k}\left(V^{\prime}\right) .
$$

Furthermore, for $\lambda \neq 0$ we have

$$
\begin{aligned}
P_{\lambda}^{\alpha} Q_{\lambda}^{\alpha}-P_{\lambda} Q_{\lambda}+\alpha \operatorname{Id}_{W_{\lambda}} & =\operatorname{Id}_{V_{\lambda}} \otimes \operatorname{diag}\left(\alpha, \cdots, \alpha,\left(1-d_{\lambda}\right) \alpha\right) \\
& =\left[N_{\lambda}, X_{\lambda}\right],
\end{aligned}
$$


where

$$
X_{\lambda}:=\operatorname{Id}_{V_{\lambda}} \otimes\left(\begin{array}{cccccc}
0 & & \cdots & & 0 \\
\alpha & 0 & & & \\
0 & 2 \alpha & \ddots & & \vdots \\
\vdots & \ddots & \ddots & 0 & \\
0 & \cdots & 0 & \left(d_{\lambda}-1\right) \alpha & 0
\end{array}\right)
$$

Thus we obtain

$$
P^{\alpha} Q^{\alpha}-P Q+\alpha \operatorname{Id}_{W}=\left[N, \bigoplus X_{\lambda}\right] \in \operatorname{Ker} p_{N}
$$

which implies

$$
\mu_{N}\left(Q^{\alpha}, P^{\alpha}\right)=\mu_{N}(Q, P)+p_{N}\left(\alpha \operatorname{Id}_{W}\right) \in \mathcal{O}+p_{N}\left(\alpha \operatorname{Id}_{W}\right) .
$$

The result follows.

Remark 6.13. In the above proof, fix a collection of complex numbers $\xi_{0}:=0$, $\xi_{1}, \ldots, \xi_{n}$ satisfying

$$
\left(\Gamma_{0}-\xi_{0} \operatorname{Id}_{V_{0}}\right)\left(\Gamma_{0}-\xi_{1} \operatorname{Id}_{V_{0}}\right) \cdots\left(\Gamma_{0}-\xi_{n} \operatorname{Id}_{V_{0}}\right)=0 .
$$

Then it is well-known [15, §2] that the numbers

$$
\operatorname{rank}\left[\left(\Gamma_{0}-\xi_{0} \operatorname{Id}_{V_{0}}\right) \cdots\left(\Gamma_{0}-\xi_{i} \operatorname{Id}_{V_{0}}\right)\right], \quad i=0, \ldots, n
$$

characterize the conjugacy class of $\Gamma_{0}$. Now consider the matrix $P_{0} Q_{0}$, which is by definition the endomorphism of $W_{0}=V_{0} / \operatorname{Ker} \Gamma_{0} \simeq \operatorname{Im} \Gamma_{0}$ induced from $\Gamma_{0}$. It satisfies

$$
\begin{aligned}
\operatorname{rank} \prod_{j=1}^{i}\left(P_{0} Q_{0}-\xi_{j} \operatorname{Id}_{W_{0}}\right) & =\operatorname{rank} \prod_{j=1}^{i}\left(\left.\Gamma_{0}\right|_{\operatorname{Im} \Gamma_{0}}-\xi_{j} \operatorname{Id}_{\operatorname{Im} \Gamma_{0}}\right) \\
& =\operatorname{rank} \prod_{j=0}^{i}\left(\Gamma_{0}-\xi_{j} \operatorname{Id}_{V_{0}}\right), \quad i=1,2, \ldots, n .
\end{aligned}
$$

Hence the matrix $P_{0}^{\alpha} Q_{0}^{\alpha}=P_{0} Q_{0}-\alpha \operatorname{Id}_{W_{0}}$ satisfies

$$
\operatorname{rank} \prod_{j=1}^{i}\left(P_{0}^{\alpha} Q_{0}^{\alpha}+\alpha \operatorname{Id}_{W_{0}}-\xi_{j} \operatorname{Id}_{W_{0}}\right)=\operatorname{rank} \prod_{j=0}^{i}\left(\Gamma_{0}-\xi_{j} \operatorname{Id}_{V_{0}}\right), \quad i=1,2, \ldots, n .
$$

Since $P_{0}^{\alpha}$ is surjective and $Q_{0}^{\alpha}$ is injective, the above implies the following equalities for the matrix $\Gamma_{0}^{\alpha}:=Q_{0}^{\alpha} P_{0}^{\alpha}$ :

$$
\begin{gathered}
\operatorname{rank} \Gamma_{0}^{\alpha}=\operatorname{dim} W_{0}=\operatorname{rank} \Gamma_{0}, \\
\operatorname{rank}\left[\Gamma_{0}^{\alpha} \prod_{j=1}^{i}\left(\Gamma_{0}^{\alpha}+\alpha \operatorname{Id}_{V_{0}^{\prime}}-\xi_{j} \operatorname{Id}_{V_{0}^{\prime}}\right)\right]=\operatorname{rank} \prod_{j=0}^{i}\left(\Gamma_{0}-\xi_{j} \operatorname{Id}_{V_{0}}\right), \quad i=1,2, \ldots, n,
\end{gathered}
$$


which characterize the conjugacy class of $\Gamma_{0}^{\alpha}$. Therefore the normal form given by (6.2) can be computed from that contained in $\Phi_{N}\left(\mu_{N}^{-1}(O)_{V}^{N \text {-st }} / G_{N}\right)$ and $\alpha, V^{\prime}$. Note that in the extreme case $\Gamma_{0}=0$, the first equality in the above implies $\Gamma_{0}^{\alpha}=0$.

Proposition 6.14. Suppose that $(V, A) \in{ }_{\vec{l}} \mathcal{D}_{\vec{k}}$ and $\alpha \in \mathcal{E}_{\vec{l}}(\mathbb{C})$ satisfy all the assumptions in Corollary 6.4 and additionally that $(V, A)$ has a normal form at some $t \in D$. Then the middle convolution $m c_{\alpha}(V, A)$ also has a normal form at the same $t$ with the same nonzero spectra.

Proof. Set $(V, W, S, T, Q, P):=\kappa(V, A)$ and

$$
\left(W, V^{\prime}, T, S^{\prime}, P^{\prime}, Q^{\prime}\right):=\kappa \circ a d d_{\alpha} \circ \operatorname{HD}(V, A) .
$$

By definition, we have $m c_{\alpha}(V, A)=\left(V^{\prime}, S^{\prime}+Q^{\prime}\left(z \operatorname{Id}_{W}-T\right)^{-1} P^{\prime}\right)$ and

$$
P\left(\zeta \operatorname{Id}_{V}-S\right)^{-1} Q+\alpha(\zeta) \operatorname{Id}_{W}=P^{\prime}\left(\zeta \operatorname{Id}_{V^{\prime}}-S^{\prime}\right)^{-1} Q^{\prime}
$$

Taking the residue at $\zeta=\infty$ on both sides, we obtain

$$
-P Q+\operatorname{Res}_{\zeta=\infty} \alpha(\zeta) \operatorname{Id}_{W}=-P^{\prime} Q^{\prime}
$$

and further taking the projection $\mathfrak{g l}(W) \rightarrow \mathfrak{g l}\left(W_{t}\right) \stackrel{p_{N_{t}}}{\longrightarrow} \mathfrak{g}_{N_{t}}^{*}$ on both sides, we obtain

$$
\mu_{N_{t}}\left(Q_{t}, P_{t}\right)+p_{N_{t}}\left(\operatorname{Res}_{\zeta=\infty} \alpha(\zeta) \operatorname{Id}_{W_{t}}\right)=\mu_{N_{t}}\left(Q_{t}^{\prime}, P_{t}^{\prime}\right)
$$

where $Q_{t}, P_{t}, Q_{t}^{\prime}, P_{t}^{\prime}$ are the block components of $Q, P, Q^{\prime}, P^{\prime}$ relative to the decomposition $W=\bigoplus_{t} W_{t}$ as usual. Set $\beta:=\operatorname{Res}_{\zeta=\infty} \alpha(\zeta)$ and let $O$ be the $G_{N_{t}}$-coadjoint orbit through $\mu_{N_{t}}\left(Q_{t}, P_{t}\right)$. Then the above relation implies that the set

$$
\mu_{N_{t}}^{-1}\left(O+p_{N_{t}}\left(\beta \operatorname{Id}_{W}\right)\right)_{V^{\prime}}^{N_{t} \text {-st }} / G_{N_{t}}
$$

is non-empty, because $m c_{\alpha}(V, A)$ is irreducible and hence $\left(Q_{t}^{\prime}, P_{t}^{\prime}\right)$ gives a point in the above by Lemma 5.5. Since $O, V, V^{\prime}$ and $\beta$ satisfy all the conditions in Lemma 6.12, the image of the above set under $\Phi_{N_{t}}$ contains a normal form which has the same nonzero spectra as that in $\Phi_{N_{t}}\left(\mu_{N_{t}}^{-1}(O)_{V}^{N_{t} \text {-st }} / G_{N_{t}}\right)$.

Remark 6.15. In the situation of Proposition 6.14, the proof of Lemma 6.12, more specifically (6.2), together with Remark 6.13 enables us to compute the normal form of $m c_{\alpha}(V, A)$ from that of $(V, A)$ and the rank of $m c_{\alpha}(V, A)$. 
Remark 6.16. Suppose that $(V, A)$ and $\alpha$ satisfy all the assumptions in Corollary 6.4 and additionally $(V, A)$ has a normal form at $\infty$ (see Remark 6.10). Then it is easy to see that $m c_{\alpha}(V, A)$ has a normal form at $\infty$ if and only if $\alpha$ is Fuchsian. Moreover, in this case, the normal form can be computed as follows. Let us use the same notation here as in the above proof and let $V=\bigoplus_{s \in E} V_{s}, V^{\prime}=\bigoplus_{s \in E} V_{s}^{\prime}$ be the eigenspace decomposition for $S, S^{\prime}$ respectively. Then writing $\alpha(\zeta)=\sum_{s \in E}(\zeta-s)^{-1} \alpha_{s}$, we have

$$
P_{s} Q_{s}+\alpha_{s} \operatorname{Id}_{W}=P_{s}^{\prime} Q_{s}^{\prime}, \quad s \in E,
$$

where $Q_{s}, P_{s}, Q_{s}^{\prime}, P_{s}^{\prime}$ are the block components of $Q, P, Q^{\prime}, P^{\prime}$ relative to the decomposition $V=\bigoplus_{s} V_{s}, V^{\prime}=\bigoplus_{s} V_{s}^{\prime}$. Set $\Omega_{s}:=Q_{s} P_{s}, \Omega_{s}^{\prime}:=Q_{s}^{\prime} P_{s}^{\prime}$. Then the normal forms of $(V, A)$ and $m c_{\alpha}(V, A)$ at $\infty$ are described as

$$
\bigoplus_{s \in E}\left(-s w^{-2} \operatorname{Id}_{V_{s}}-\Omega_{s} w^{-1}\right), \quad \bigoplus_{s \in E}\left(-s w^{-2} \operatorname{Id}_{V_{s}}-\Omega_{s}^{\prime} w^{-1}\right) .
$$

Note that $P_{s}, P_{s}^{\prime}$ are injective and $Q_{s}, Q_{s}^{\prime}$ are surjective because the data $(W, V, S, P, Q)$ and $\left(W, V^{\prime}, S^{\prime}, P^{\prime}, Q^{\prime}\right)$ are both stable. Fix $s \in E$ and let $\eta_{1}, \ldots, \eta_{m}$ be complex numbers satisfying

$$
\left(\Omega_{s}-\eta_{1} \operatorname{Id}_{V_{s}}\right) \cdots\left(\Omega_{s}-\eta_{m} \operatorname{Id}_{V_{s}}\right)=0 .
$$

Set $\eta_{0}:=0$. Then an argument similar to that in Remark6.13 shows

$$
\begin{aligned}
\operatorname{dim} V_{s} & =\operatorname{rank} P_{s} Q_{s}=\operatorname{rank}\left(P_{s}^{\prime} Q_{s}^{\prime}-\alpha_{s} \operatorname{Id}_{W}\right), \\
\operatorname{rank} \prod_{j=1}^{i}\left(\Omega_{s}-\eta_{j} \operatorname{Id}_{W}\right) & =\operatorname{rank} \prod_{j=0}^{i}\left(P_{s} Q_{s}-\eta_{j} \operatorname{Id}_{W}\right) \\
& =\operatorname{rank} \prod_{j=0}^{i}\left(P_{s} Q_{s}-\alpha_{s} \operatorname{Id}_{W}-\eta_{j} \operatorname{Id}_{W}\right), \quad i=1,2, \ldots, m .
\end{aligned}
$$

These characterize the conjugacy class of $P_{s}^{\prime} Q_{s}^{\prime}$. If none of $\eta_{i}^{\prime}:=\alpha_{s}+\eta_{i}, i=0, \ldots, m$ are zero, $P_{s}^{\prime} Q_{s}^{\prime}$ is invertible and hence the above equalities characterize the matrix $\Omega_{s}^{\prime}$ also. If there is some $l$ such that $\eta_{l}^{\prime}=0$, then for $i=0,1, \ldots, m$, we have

$$
\begin{aligned}
\operatorname{rank} \prod_{\substack{j \neq l, 0 \leq j \leq i}}\left(\Omega_{s}^{\prime}-\eta_{j}^{\prime} \operatorname{Id}_{V_{s}^{\prime}}\right) & =\operatorname{rank}\left[\left.\prod_{\substack{j \neq l, 0 \leq j \leq i}}\left(P_{s} Q_{s}-\eta_{j}^{\prime} \operatorname{Id}_{W}\right)\right|_{\left.\operatorname{Im} P_{s} Q_{s}\right]}\right. \\
& = \begin{cases}\operatorname{rank}\left[P_{s} Q_{s} \prod_{j=0}^{i}\left(P_{s} Q_{s}-\eta_{j}^{\prime} \operatorname{Id}_{W}\right)\right] & i<l, \\
\operatorname{rank} \prod_{j=0}^{i}\left(P_{s} Q_{s}-\eta_{j}^{\prime} \operatorname{Id}_{W}\right) & i \geq l,\end{cases}
\end{aligned}
$$

which characterizes the conjugacy class of $\Omega_{s}^{\prime}$. 
Remark 6.17. We can further generalize the middle convolution. Let us recall the category $\mathcal{D}$ introduced in Remark 5.14. For any rank 1 object $(\mathbb{C}, \alpha) \in \mathcal{D}$, we can define the addition $a d d_{\alpha}$, and hence the middle convolution $m c_{\alpha}=\mathrm{HD} \circ a d d_{\alpha} \circ \mathrm{HD}$ with $\alpha$, as endo-functors of $\mathcal{D}$. However decomposing the parameter as $\alpha(\zeta)=a+\alpha^{0}(\zeta)$, where $a:=\lim _{\zeta=\infty} \alpha(\zeta)$, we can easily see that the first component $a$ just plays the role of a translation of coordinate $\zeta$ in the middle convolution; $m c_{\alpha}(V, A)$ is obtained from $m c_{\alpha^{0}}(V, A)$ by the coordinate change $\zeta \mapsto \zeta-a$. Therefore only the case $\alpha(\zeta)=\alpha^{0}(\zeta) \in \mathcal{E}_{\vec{l}}(\mathbb{C})$ for some $E, \vec{l}$ is essential.

\section{Generalized Katz's algorithm}

In this section we generalize Katz's algorithm as an application of our middle convolution. Hereafter we assume $E=\{0\}$ and $\vec{l}=(1)$. Hence an object of the category $\vec{D}_{\vec{k}}$ is just a pair $(V, A)$ of a finite-dimensional $\mathbb{C}$-vector space $V$ and a system $A(z) \in \mathcal{E}_{\vec{k}}(V)$. We denote by $\mathcal{D}_{\vec{k}}^{0}$ the full subcategory of ${ }_{\vec{l}} \mathcal{D}_{\vec{k}}$ consisting of objects $(V, A)$ satisfying $\operatorname{Res}_{z=\infty} A(z)=0$, namely, that $A(z)$ has no singularity at $\infty$. For $(V, A) \in \mathcal{D}_{\vec{k}}^{0}$, we denote by $\mathbb{O}(A) \subset \mathcal{E}_{\vec{k}}(V)$ the $G_{\vec{k}}(V)$-coadjoint orbit through $A(z)$. Note that if $(V, A)$ is irreducible, then it represents a point in the space $\mathfrak{M}^{\mathrm{irr}}(\mathbb{O}(A), 0)$.

Definition 7.1. An irreducible pair $(V, A) \in \mathcal{D}_{\vec{k}}^{0}$ is said to be naively rigid if the space $\mathfrak{M}^{\mathrm{irr}}(\mathbb{O}(A), 0)$ consists of only one point.

Note that the dimension of $\mathfrak{M}^{\text {irr }}(\mathbb{O}, 0)$ can be computed (if it is nonempty) as

$$
\operatorname{dim} \mathfrak{M}^{\mathrm{irr}}(\mathbb{O}, 0)=\operatorname{dim} \mathbb{O}-2 \operatorname{dim} \operatorname{GL}(V)+2,
$$

because $\mathfrak{M}^{\text {irr }}(\mathbb{O}, 0)$ is an open subset of the symplectic quotient of $\mathbb{O}$ by the action of $\operatorname{GL}(V)$ which acts on irreducible systems with trivial stabilizer $\mathbb{C}^{\times}$by Schur's lemma.

Remark 7.2. The reason why we say 'naively rigid', not just 'rigid', is the following. Katz originally said an irreducible local system on $X:=\mathbb{P}^{1} \backslash D$ to be rigid if it is determined up to isomorphism by its local monodromies around the points in $D$. A natural generalization of Katz's rigidity to the irregular singular case was introduced by Bloch-Esnault [9], who said an irreducible algebraic connection on $X$ to be rigid if it is determined up to isomorphism by its formal (meromorphic) type on $D$. On the other hand, in the above we consider the truncated formal type $\mathbb{O}(A)$, not the formal meromorphic type.

Nevertheless, in a good situation, there is an implication between the two notions. Suppose that a pair $(V, A) \in \mathcal{D}_{\vec{k}}^{0}$ has a reduced normal form $\Lambda_{t}(z)=\sum \Lambda_{t, i} z^{-i}$ (see 
Remark 6.11) at each $t \in D$. Then $\operatorname{Res}_{z=\infty} A(z)=0$ implies

$$
\sum_{t \in D} \operatorname{tr} \Lambda_{t, 1}=\sum_{t \in D} \operatorname{tr} \operatorname{Res}_{z=t} A(z)=0
$$

since $\operatorname{tr} \operatorname{Res}_{z=0}: \mathfrak{g}_{k}^{*}(V) \rightarrow \mathbb{C}$ is $G_{k}(V)$-invariant. Let $\alpha_{t}^{(1)}, \ldots, \alpha_{t}^{(n)}$ be the eigenvalues of $\Lambda_{t, 1}$ repeated according to multiplicities (so $n=\operatorname{dim} V$ ). We assume that any collection $\left(I_{t}\right)_{t \in D}$ of nonempty proper sub-index sets $I_{t} \subset\{1, \ldots, n\}$ of the same cardinality satisfies

$$
\sum_{t \in D} \sum_{i \in I_{t}} \alpha_{t}^{(i)} \notin \mathbb{Z}
$$

This assumption implies that the trivial bundle $\mathcal{V}:=X \times V$ together with the algebraic connection $\nabla_{A}:=d-A(z) d z$ is irreducible (and hence that the pair $(V, A)$ is irreducible). Indeed, suppose that a subbundle of $\mathcal{V}$ preserved by $\nabla_{A}$ is given. A trivialization of it then gives an algebraic connection $\nabla_{B}=d-B(z) d z$ on a trivial bundle $\mathcal{W}=X \times W$ together with an embedding $\phi:\left(\mathcal{W}, \nabla_{B}\right) \hookrightarrow\left(\mathcal{V}, \nabla_{A}\right)$. Note that by the assumption and Remark 6.11, the connection $\nabla_{A}$ is unramified at any $t \in D$. Therefore an argument similar to that in [ 6 , Theorem 6.4] shows that at any $t \in D$, the connection $\nabla_{B}$ is also unramified and its reduced normal form is given by the restriction of $\Lambda_{t}$ on some subspace of $V$ (which has the same dimension as $W$ ). Thus we obtain a subset $I_{t} \subset\{1, \ldots, n\}$ of cardinality $\operatorname{dim} W$, which indexes the eigenvalues of the reduced normal form of $\nabla_{B}$ at $t$ (repeated according to multiplicities). Now using the 'polar decomposition'

$$
\operatorname{Aut}_{\mathcal{F}}\left(\mathbb{C}^{m} \otimes \mathcal{F}\right)=\operatorname{Aut}_{\mathbb{C}[z]]}\left(\mathbb{C}^{m} \otimes \mathbb{C}[[z]]\right) \cdot G \cdot \operatorname{Aut}_{\mathbb{C}[[z]]}\left(\mathbb{C}^{m} \otimes \mathbb{C}[[z]]\right),
$$

where

$$
G=\left\{\operatorname{diag}\left(z^{r_{1}}, \ldots, z^{r_{m}}\right) \mid r_{i} \in \mathbb{Z}, r_{1} \leq \cdots \leq r_{m}\right\}
$$

we have

$$
0=\operatorname{tr} \operatorname{Res}_{z=\infty} B(z)=-\sum_{t \in D} \operatorname{tr} \operatorname{Res}_{z=t} B(z) \in-\sum_{t \in D} \sum_{i \in I_{t}} \alpha_{t}^{(i)}+\mathbb{Z} .
$$

The assumption implies $W=0$ or $W=V$. Hence $\left(\mathcal{V}, \nabla_{A}\right)$ is irreducible.

Under those assumptions, we can show that if $\left(\mathcal{V}, \nabla_{A}\right)$ is rigid, then the pair $(V, A)$ is naively rigid, as follows. Let $\left(V, A^{\prime}\right) \in \mathcal{D}_{\vec{k}}^{0}$ be an irreducible pair satisfying $\mathbb{O}\left(A^{\prime}\right)=$ $O(A)$. Then by Remark 6.11, the $\mathcal{E}_{k_{t}}(V)$-component $A_{t}^{\prime}$ of $A^{\prime}$ is equivalent to $\Lambda_{t}$ under the $\operatorname{Aut}_{\mathbb{C}[z]]}(V \otimes \mathbb{C}[[z]])$-action for any $t \in D$. On the other hand, the above argument shows that the algebraic connection $\left(\mathcal{V}, \nabla_{A^{\prime}}\right)$ is irreducible. Hence by the rigidity, there is an isomorphism $\left(\mathcal{V}, \nabla_{A^{\prime}}\right) \simeq\left(\mathcal{V}, \nabla_{A}\right)$, which gives for each $t \in D$ an element of $\operatorname{Aut}_{\mathcal{F}}(V \otimes \mathcal{F})$ connecting $A_{t}^{\prime}$ and the $\mathcal{E}_{k_{t}}(V)$-component $A_{t}$ of $A$. Here, because $A_{t}$ and 
$A_{t}^{\prime}$ are both equivalent to $\Lambda_{t}$ under the $\operatorname{Aut}_{\mathbb{C}[z]]}(V \otimes \mathbb{C}[[z]])$-action, [6, Theorem 7.2] implies that it is in fact an element of $\operatorname{Aut}_{\mathbb{C}[z]]}(V \otimes \mathbb{C}[[z]])$. Hence the isomorphism extends to a holomorphic bundle isomorphism $\mathbb{P}^{1} \times V \rightarrow \mathbb{P}^{1} \times V$, which must be constant and hence gives $\left(V, A^{\prime}\right) \sim(V, A)$.

Recall that any element $A(z) \in \mathcal{E}_{k}(V)$ defines a matrix $\widehat{A} \in \operatorname{End}\left(V^{\oplus k}\right)$ by (4.2). Then the key lemma is the following:

Lemma 7.3. For any element $A(z) \in \mathcal{E}_{k}(V)$ having a normal form, there exists $\alpha(z) \in$ $\mathcal{E}_{k}(\mathbb{C})$ such that

$$
\operatorname{dim} Z(A) \leq(\operatorname{dim} V) \operatorname{dim} \operatorname{Ker}\left(\widehat{A}-\widehat{\alpha \operatorname{Id}_{V}}\right),
$$

where $Z(A)$ denotes the stabilizer of $A(z)$ in $G_{k}(V)$.

Proof. Since $\operatorname{dim} Z(A)=\operatorname{dim} Z(g \cdot A)$ for any $g(z) \in G_{k}(V)$, we may assume that $A(z)$ itself is a normal form;

$$
A(z)=\bigoplus_{\lambda \in \Sigma}\left(\lambda(z) \operatorname{Id}_{V_{\lambda}}+\frac{\Gamma_{\lambda}}{z}\right)=\sum_{i=1}^{k} \Lambda_{i} z^{-i}
$$

Then a slight modification of the argument in the proof of Proposition 6.8 shows that an element $g(z)=\sum_{i} g_{i} z^{i} \in G_{k}(V)$ stabilizes $A(z)$ if and only if

$$
g_{i-1} \in \mathfrak{h}_{i}:=\operatorname{Ker}\left(\operatorname{ad}_{\Lambda_{i}}\right) \cap \cdots \cap \operatorname{Ker}\left(\operatorname{ad}_{\Lambda_{k}}\right), \quad i=1,2, \ldots, k
$$

Hence $\operatorname{dim} Z(A)=\sum_{i=1}^{k} \operatorname{dim} \mathfrak{h}_{i}$. Similarly, one can easily see that $v_{i} \in V, i=1, \ldots, k$ satisfy the equation

$$
\left(\begin{array}{cccc}
\Lambda_{k} & \Lambda_{k-1} & \cdots & \Lambda_{1} \\
& \Lambda_{k} & \ddots & \vdots \\
& & \ddots & \Lambda_{k-1} \\
0 & & & \Lambda_{k}
\end{array}\right)\left(\begin{array}{c}
v_{k} \\
v_{k-1} \\
\vdots \\
v_{1}
\end{array}\right)=0
$$

if and only if

$$
v_{i} \in \operatorname{Ker} \Lambda_{i} \cap \cdots \cap \operatorname{Ker} \Lambda_{k}, i=1,2, \ldots, k .
$$

Let $V\left(\alpha_{i}, \ldots, \alpha_{k}\right)$ be the simultaneous eigenspace for $\left(\Lambda_{i}, \ldots, \Lambda_{k}\right)$ with eigenvalue $\left(\alpha_{i}, \ldots, \alpha_{k}\right)$. Replacing $\Lambda$ with $\Lambda-\alpha \operatorname{Id}_{V}$ in the above equation, we then obtain

$$
\operatorname{dim} \operatorname{Ker}\left(\widehat{A}-\widehat{\alpha \operatorname{Id}_{V}}\right)=\sum_{i=1}^{k} \operatorname{dim} V\left(\alpha_{i}, \ldots, \alpha_{k}\right) .
$$


On the other hand, we have

$$
\mathfrak{h}_{i}=\bigoplus_{\left(\alpha_{i}, \ldots, \alpha_{k}\right)} \mathfrak{g l}\left(V\left(\alpha_{i}, \ldots, \alpha_{k}\right)\right), \quad i \geq 2
$$

To describe $\operatorname{dim} \mathfrak{h}_{1}$ analogously, we apply the fact mentioned in Remark 4.4 to the matrix $\Gamma_{\lambda}$ for each $\lambda=\sum_{i \geq 2} \lambda_{i} z^{-i} \in \Sigma$. For any eigenvalue $\lambda_{1} \in \mathbb{C}$ of $\Gamma_{\lambda}$, there exist a decomposition of the corresponding generalized eigenspace

$$
\operatorname{Ker}\left(\Gamma_{\lambda}-\lambda_{1} \operatorname{Id}_{V_{\lambda}}\right)^{\operatorname{dim} V_{\lambda}}=\bigoplus_{j=1}^{r_{\lambda_{1}, \lambda_{2}, \ldots, \lambda_{k}}} V^{j}\left(\lambda_{1}, \lambda_{2}, \ldots, \lambda_{k}\right)
$$

with $V^{1}\left(\lambda_{1}, \lambda_{2}, \ldots, \lambda_{k}\right)=V\left(\lambda_{1}, \lambda_{2}, \ldots, \lambda_{k}\right)$ and injections

$$
\iota: V^{j}\left(\lambda_{1}, \lambda_{2}, \ldots, \lambda_{k}\right) \hookrightarrow V^{j-1}\left(\lambda_{1}, \lambda_{2}, \ldots, \lambda_{k}\right), \quad j=2, \ldots, r_{\lambda_{1}, \ldots, \lambda_{k}}
$$

such that the matrix $\Gamma_{\lambda}-\lambda_{1} \operatorname{Id}_{V_{\lambda}}$ restricted on $\operatorname{Ker}\left(\Gamma_{\lambda}-\lambda_{1} \operatorname{Id}_{V_{\lambda}}\right)^{\operatorname{dim} V_{\lambda}}$ is written as (4.1). Then we can compute [38] the dimension of the centralizer $Z\left(\Gamma_{\lambda}\right)$ as

$$
\operatorname{dim} Z\left(\Gamma_{\lambda}\right)=\sum_{\lambda_{1}} \sum_{j=1}^{r_{\lambda_{1}, \lambda_{2}, \ldots, \lambda_{k}}}\left(\operatorname{dim} V^{j}\left(\lambda_{1}, \lambda_{2}, \ldots, \lambda_{k}\right)\right)^{2}
$$

whence

$$
\operatorname{dim} \mathfrak{h}_{1}=\sum_{\lambda \in \Sigma} \operatorname{dim} Z\left(\Gamma_{\lambda}\right)=\sum_{\left(\lambda_{1}, \ldots, \lambda_{k}\right)} \sum_{j=1}^{r_{\lambda_{1}, \lambda_{2}, \ldots, \lambda_{k}}}\left(\operatorname{dim} V^{j}\left(\lambda_{1}, \lambda_{2}, \ldots, \lambda_{k}\right)\right)^{2} .
$$

Thus writing $V^{j}\left(\alpha_{1}, \ldots, \alpha_{k}\right) \equiv V^{j}(\alpha)$ with $\alpha=\sum_{i=1}^{k} \alpha_{i} z^{-i}$, we obtain

$$
\operatorname{dim} Z(A)=\sum_{\alpha} \sum_{j}\left(\operatorname{dim} V^{j}(\alpha)\right)^{2}+\sum_{i=2}^{k} \sum_{\left(\alpha_{i}, \ldots, \alpha_{k}\right)}\left(\operatorname{dim} V\left(\alpha_{i}, \ldots, \alpha_{k}\right)\right)^{2} .
$$

Now we prove the lemma. First we rewrite (7.1) as

$$
\begin{aligned}
\operatorname{dim} \operatorname{Ker}\left(\widehat{A}-\widehat{\alpha \operatorname{Id}_{V}}\right) & =\operatorname{dim} V(\alpha)+\sum_{i=2}^{k} \sum_{\left(\beta_{1}, \ldots, \beta_{i-1}\right)} \sum_{j} \operatorname{dim} V^{j}\left(\beta_{1}, \ldots, \beta_{i-1}, \alpha_{i}, \ldots, \alpha_{k}\right) \\
& =\operatorname{dim} V(\alpha)+\sum_{i=2}^{k} \sum_{\beta} \sum_{j} \delta_{\alpha_{i}, \beta_{i}} \cdots \delta_{\alpha_{k}, \beta_{k}} \operatorname{dim} V^{j}(\beta),
\end{aligned}
$$

where $\delta_{\alpha_{i}, \beta_{i}}$ denotes Kronecker's delta symbol. Setting

$$
\delta(\alpha, \beta ; j):=\delta_{j, 1} \delta_{\alpha, \beta}+\sum_{i=2}^{k} \delta_{\alpha_{i}, \beta_{i}} \cdots \delta_{\alpha_{k}, \beta_{k}}, \quad \alpha=\sum_{i=1}^{k} \alpha_{i} z^{-i}, \beta=\sum_{i=1}^{k} \beta_{i} z^{-i}
$$


we can describe the above equality as

$$
\operatorname{dim} \operatorname{Ker}\left(\widehat{A}-\widehat{\alpha \operatorname{Id}_{V}}\right)=\sum_{\beta, j} \delta(\alpha, \beta ; j) \operatorname{dim} V^{j}(\beta) .
$$

Next we rewrite (7.2) as

$$
\begin{aligned}
\operatorname{dim} Z(A)= & \sum_{\alpha} \sum_{j}\left(\operatorname{dim} V^{j}(\alpha)\right)^{2}+\sum_{i=2}^{k} \sum_{\left(\alpha_{i}, \ldots, \alpha_{k}\right)}\left(\sum_{\beta} \sum_{j} \delta_{\alpha_{i}, \beta_{i}} \cdots \delta_{\alpha_{k}, \beta_{k}} \operatorname{dim} V^{j}(\beta)\right)^{2} \\
= & \sum_{\alpha} \sum_{j}\left(\operatorname{dim} V^{j}(\alpha)\right)^{2} \\
& \quad+\sum_{i=2}^{k} \sum_{\left(\alpha_{i}, \ldots, \alpha_{k}\right)} \sum_{\beta, \gamma} \sum_{j, l} \delta_{\alpha_{i}, \beta_{i}} \cdots \delta_{\alpha_{k}, \beta_{k}} \delta_{\alpha_{i}, \gamma_{i}} \cdots \delta_{\alpha_{k}, \gamma_{k}} \operatorname{dim} V^{j}(\beta) \operatorname{dim} V^{l}(\gamma) \\
= & \sum_{\alpha} \sum_{j}\left(\operatorname{dim} V^{j}(\alpha)\right)^{2}+\sum_{i=2}^{k} \sum_{\beta, \gamma} \sum_{j, l} \delta_{\beta_{i}, \gamma_{i}} \cdots \delta_{\beta_{k}, \gamma_{k}} \operatorname{dim} V^{j}(\beta) \operatorname{dim} V^{l}(\gamma) .
\end{aligned}
$$

Using the inequality $\operatorname{dim} V^{j}(\alpha) \leq \operatorname{dim} V(\alpha)$, we obtain

$$
\begin{aligned}
\operatorname{dim} Z(A) \leq & \sum_{\alpha} \sum_{j} \operatorname{dim} V^{j}(\alpha) \operatorname{dim} V(\alpha) \\
& +\sum_{i=2}^{k} \sum_{\beta, \gamma} \sum_{j, l} \delta_{\beta_{i}, \gamma_{i}} \cdots \delta_{\beta_{k}, \gamma_{k}} \operatorname{dim} V^{j}(\beta) \operatorname{dim} V^{l}(\gamma) \\
= & \sum_{\beta, \gamma} \sum_{j, l}\left(\delta_{l, 1} \delta_{\beta, \gamma}+\sum_{i=2}^{k} \delta_{\beta_{i}, \gamma_{i}} \cdots \delta_{\beta_{k}, \gamma_{k}}\right) \operatorname{dim} V^{j}(\beta) \operatorname{dim} V^{l}(\gamma) \\
= & \sum_{\beta, \gamma} \sum_{j, l} \delta(\beta, \gamma ; l) \operatorname{dim} V^{j}(\beta) \operatorname{dim} V^{l}(\gamma) \\
= & \sum_{\beta, j} \operatorname{dim} \operatorname{Ker}\left(\widehat{A}-\widehat{\beta \operatorname{Id}_{V}}\right) \operatorname{dim} V^{j}(\beta) .
\end{aligned}
$$

Take $\alpha \in \mathcal{E}_{k}(\mathbb{C})$ to attain the maximum of the values $\operatorname{dim} \operatorname{Ker}\left(\widehat{A}-\widehat{\alpha \operatorname{Id}_{V}}\right)$. Then

$$
\operatorname{dim} Z(A) \leq \sum_{\beta, j} \operatorname{dim} \operatorname{Ker}\left(\widehat{A}-\widehat{\alpha \operatorname{Id}_{V}}\right) \operatorname{dim} V^{j}(\beta)=\operatorname{dim} \operatorname{Ker}\left(\widehat{A}-\widehat{\alpha \operatorname{Id}_{V}}\right) \operatorname{dim} V
$$

which is the desired inequality.

Lemma 7.4. Suppose that a pair $(V, A) \in \mathcal{D}_{\vec{k}}^{0}$ is irreducible and $(V, A) \nsim(\mathbb{C}, 0)$. Then the rank of $\operatorname{HD}(V, A)$ is greater than or equal to $2 \operatorname{dim} V$.

Proof. By the assumption and Lemma 5.6, $(V, W, 0, T, Q, P):=\kappa(V, A)$ is irreducible. Since the pairs $(\operatorname{Im} Q, W)$ and $(\operatorname{Ker} P, 0)$ are both subrepresentations of it, we see that 
$Q$ is surjective and $P$ is injective if $W \neq 0$, in other words, if $(V, A) \times(\mathbb{C}, 0)$ (see Corollary 5.9]. On the other hand, we have $Q P=-\operatorname{Res}_{z=\infty} A(z)=0$. Thus under the assumption $(V, A) \times(\mathbb{C}, 0)$, we obtain

$$
\operatorname{dim} V=\operatorname{rank} P \leq \operatorname{dim} \operatorname{Ker} Q=\operatorname{dim} W-\operatorname{dim} V .
$$

Theorem 7.5. Suppose that a pair $(V, A) \in \mathcal{D}_{\vec{k}}^{0}$ with $\operatorname{dim} V \geq 2$ is irreducible, naively rigid, and has a normal form at any $t \in D$. Then there exists a rank 1 system $\alpha(z) \in$ $\mathcal{E}_{\vec{k}}(\mathbb{C})$ such that the rank of $m c_{\lambda / \zeta}$ oadd $d_{-\alpha}(V, A)$, where $\lambda=-\operatorname{Res}_{z=\infty} \alpha(z)$, is less than $\operatorname{dim} V$.

Proof. For each $t \in D$, take $\alpha_{t}(z) \in \mathcal{E}_{k_{t}}(\mathbb{C})$ to satisfy the condition in Lemma 7.3 for $A_{t}(z)$, and set $\alpha(z):=\sum_{t \in D} \alpha_{t}(z-t)$. Let $W$ be the underlying vector space of $\mathrm{HD} \circ \operatorname{add}_{-\alpha}(V, A)$. Then, since $(V, A)$ is naively rigid, using the definition of $W$ we have

$$
\begin{aligned}
2 \operatorname{dim} \operatorname{GL}(V)-2 & =\operatorname{dim} \mathbb{O}(A) \\
& =\sum_{t \in D}\left(\operatorname{dim} G_{k_{t}}(V)-\operatorname{dim} Z\left(A_{t}\right)\right) \\
& =\operatorname{dim} \operatorname{GL}(V) \sum_{t \in D} k_{t}-\sum_{t \in D} \operatorname{dim} Z\left(A_{t}\right) \\
& \geq \operatorname{dim} V \sum_{t \in D}\left(k_{t} \operatorname{dim} V-\operatorname{dim} \operatorname{Ker}\left(\widehat{A_{t}}-\widehat{\alpha_{t} \widehat{\operatorname{Id}}_{V}}\right)\right) \\
& =\operatorname{dim} V \operatorname{dim} W .
\end{aligned}
$$

Hence

$$
\operatorname{dim} W \leq(2 \operatorname{dim} \operatorname{GL}(V)-2) / \operatorname{dim} V<2 \operatorname{dim} V .
$$

Note that the pair $\left(V, A-\alpha \operatorname{Id}_{V}\right)$ is irreducible as so is $(V, A)$. Therefore the above inequality together with Lemma 7.4 implies $\lambda=-\operatorname{Res}_{z=\infty} \alpha(z) \neq 0$. By Example 6.6, we thus have

$$
\operatorname{dim} V^{\lambda / \zeta}=\operatorname{dim} W-\operatorname{dim} V<\operatorname{dim} V .
$$

Note that the functor $a d d_{\alpha} \circ m c_{\lambda / \zeta} \circ a d d_{\alpha}$, where $\lambda=\operatorname{Res}_{z=\infty} \alpha(z)$, preserves the full subcategory $\mathcal{D}_{\vec{k}}^{0}$ for any $\alpha(z) \in \mathcal{E}_{\vec{k}}(\mathbb{C})$ by Example 6.6. Therefore the above theorem together with Proposition 6.14 implies the following: 
Corollary 7.6. Suppose that a pair $(V, A) \in \mathcal{D}_{\vec{k}}^{0}$ with $\operatorname{dim} V \geq 2$ is irreducible, naively rigid, and has a normal form at any $t \in D$. Then applying a suitable finite iteration of operations of the form

$$
a d d_{\alpha} \circ m c_{\lambda / \zeta} \circ a d d_{\alpha}, \quad \alpha \in \mathcal{E}_{\vec{k}}(\mathbb{C}), \lambda=\operatorname{Res}_{z=\infty} \alpha(z),
$$

makes $(V, A)$ into an irreducible pair of rank 1.

Example 7.7. (a) Clearly all rank 1 objects in $\mathcal{D}_{\vec{k}}^{0}$ are irreducible and naively rigid.

(b) Let $\left(\mathbb{C}^{2}, A\right) \in \mathcal{D}_{\vec{k}}^{0}$ be an irreducible rank 2 object having a normal form

$$
\Lambda_{t}(z)=\left(\begin{array}{cc}
\alpha_{t}(z) & 0 \\
0 & \beta_{t}(z)
\end{array}\right), \quad \alpha_{t}(z), \beta_{t}(z) \in \mathcal{E}_{k_{t}}(\mathbb{C})
$$

at each $t \in D$. The addition functor with $-\beta(z):=-\sum_{t \in D} \beta_{t}(z-t)$ sends $\left(\mathbb{C}^{2}, A\right)$ to $\left(\mathbb{C}^{2}, A-\beta \operatorname{Id}_{\mathbb{C}^{2}}\right)$, which has the normal form $\Lambda_{t}-\beta_{t} \operatorname{Id}_{\mathbb{C}^{2}}=\left(\alpha_{t}-\beta_{t}\right) \oplus 0$ at each $t$. Let $\left(\mathbb{C}^{2}, W_{t}, N_{t}, Q_{t}, P_{t}\right)$ be the canonical datum for $\Lambda_{t}-\beta_{t} \operatorname{Id}_{\mathbb{C}^{2}}$. As in the proof of Lemma 6.12, we then see that $W_{t} \simeq \mathbb{C} \otimes \mathbb{C}^{d_{t}}=\mathbb{C}^{d_{t}}$, where $d_{t}=\operatorname{ord}\left(\alpha_{t}-\beta_{t}\right)$, and $N_{t}$ is given by the nilpotent single Jordan block. Thus we have $G_{N_{t}} \simeq G_{d_{t}}(\mathbb{C}) \subset \mathrm{GL}\left(\mathbb{C}^{d_{t}}\right)$, which is abelian. In particular all elements in $\mathfrak{g}_{N_{t}}^{*}$ are fixed by the coadjoint action. Therefore in Theorem 4.14, the $G_{\vec{k}}(V)$-coadjoint orbit $\mathbb{O}(A)-\beta \operatorname{Id}_{\mathbb{C}^{2}}$ is described as $\mu_{T}^{-1}(O)^{T-\text { st }} / G_{T}$ with $O$ being just a single (central) element. Thus we can compute the dimension of the naive moduli space as

$$
\begin{aligned}
\operatorname{dim} \mathfrak{M}^{\operatorname{irr}}(\mathbb{O}(A), 0) & =\operatorname{dim} \mathfrak{M}^{\mathrm{irr}}\left(\mathbb{O}(A)-\beta \operatorname{Id}_{\mathbb{C}^{2}},-w^{-1} \operatorname{Res}_{z=\infty} \beta(z) \operatorname{Id}_{\mathbb{C}^{2}}\right) \\
& =2 \sum_{t \in D} \operatorname{dim} \mathbf{M}\left(\mathbb{C}^{2}, W_{t}\right)-2 \sum_{t \in D} \operatorname{dim} G_{N_{t}}-2 \operatorname{dim} \operatorname{GL}\left(\mathbb{C}^{2}\right)+2 \\
& =4 \sum_{t \in D} d_{t}-2 \sum_{t \in D} d_{t}-6 \\
& =2 \sum_{t \in D} d_{t}-6 .
\end{aligned}
$$

Now suppose that $\left(\mathbb{C}^{2}, A\right)$ is naively rigid. Then the above formula implies that the number of points $t \in D$ with $d_{t}>0$ is at most 3 , so we may assume $D=\left\{t_{1}, t_{2}, t_{3}\right\}$, and up to permutation the triple $\left(d_{t_{1}}, d_{t_{2}}, d_{t_{3}}\right)$ is one of the following:

$$
(3,0,0), \quad(2,1,0), \quad(1,1,1) .
$$

In all the cases, the operator (7.3) with $\alpha:=-\beta$ makes $\left(\mathbb{C}^{2}, A\right)$ into a rank 1 object. 
Remark 7.8. In the situation of Example 7.7, (b), the case $\operatorname{dim} \mathfrak{M}^{\mathrm{irr}}(\mathbb{O}(A), 0)=2$ is also important. In this case, the number of points $t \in D$ with $d_{t}>0$ is at most 4 , so we may assume $D=\left\{t_{1}, t_{2}, t_{3}, t_{4}\right\}$, and up to permutation the quadruple $\left(d_{t_{1}}, d_{t_{2}}, d_{t_{3}}, d_{t_{4}}\right)$ is one of the following:

$$
(4,0,0,0), \quad(3,1,0,0), \quad(2,2,0,0), \quad(2,1,1,0), \quad(1,1,1,1)
$$

Unlike in the case $\operatorname{dim} \mathfrak{M}^{\mathrm{irr}}(\mathbb{O}(A), 0)=0$, the rank of $\left(\mathbb{C}^{2}, A\right)$ does not change under the operation (7.3) with $\alpha:=-\beta$. Indeed, Example 6.6 implies that the rank of the resulting system is equal to $\sum_{t} \operatorname{dim} W_{t}-\operatorname{dim} \mathbb{C}^{2}=4-2=2$. It is well-known [35] that through the isomonodromic deformation, the above quadruples correspond to the Painlevé equations of type II, IV, III, V, VI respectively.

Acknowledgements. The author is extremely grateful to Professor Yoshishige Haraoka, Toshio Oshima and Masa-Hiko Saito for giving him many opportunities to discuss problems around the middle convolution. Also, the author is much obliged to Philip Boalch for valuable comments, and to Hiroshi Kawakami and Professor Kouichi Takemura for expounding their works to him. Kawakami's talk triggered the author's interest in the problems treated in this article. Takemura suggested in his talk a direct generalization of Dettweiler-Reiter's description of the middle convolution for irregular singular systems (in one parameter case, however systems are allowed to have a pole of arbitrary order at $\infty$ ), and conjectured that it holds basic properties of the original middle convolution. His idea is used on (4.2).

Finally, the author would like to thank the referee, who ponited out errors and a lack of argument in the earlier version of this article.

\section{References}

[1] Adams, M. R., Harnad, J., Hurtubise, J.: Dual moment maps into loop algebras. Lett. Math. Phys. 20, 299-308 (1990)

[2] Adams, M. R., Harnad, J., Hurtubise, J.: Isospectral Hamiltonian flows in finite and infinite dimensions. II. Integration of flows. Comm. Math. Phys. 134, 555-585 (1990)

[3] Adams, M. R., Harnad, J., Previato, E.: Isospectral Hamiltonian flows in finite and infinite dimensions. I. Generalized Moser systems and moment maps into loop algebras. Comm. Math. Phys. 117, 451-500 (1988)

[4] Arinkin, D.: Fourier transform and middle convolution for irregular $\mathcal{D}$-modules. arXiv.0808.0699

[5] Arinkin, D.: Rigid irregular connections on $\mathbb{P}^{1}$. arXiv:0808.0742

[6] Babbitt, D. G., Varadarajan, V. S.: Formal reduction theory of meromorphic differential equations: a group theoretic view. Pacific J. Math. 109, 1-80 (1983) 
[7] Babbitt, D. G., Varadarajan, V. S.: Local moduli for meromorphic differential equations. Astérisque 169-170, 1-217 (1989)

[8] Balser, W., Jurkat, W. B., Lutz, D. A.: On the reduction of connection problems for differential equations with an irregular singular point to ones with only regular singularities. I. SIAM J. Math. Anal. 12, 691-721 (1981)

[9] Bloch, S., Esnault, H.: Local Fourier transforms and rigidity for $\mathcal{D}$-modules. Asian J. Math. 8, 587-605 (2004)

[10] Boalch, P.: Symplectic manifolds and isomonodromic deformations. Adv. Math. 163, 137-205 (2001)

[11] Boalch, P.: From Klein to Painlevé via Fourier, Laplace and Jimbo. Proc. London Math. Soc. (3) 90, 167-208 (2005)

[12] Boalch, P.: Quivers and difference Painlevé equations. In: Groups and symmetries, pp. 25-51. CRM Proc. Lecture Notes, vol. 47. Amer. Math. Soc., Providence (2009)

[13] Boalch, P.: Irregular connections and Kac-Moody root systems. arXiv:0806.1050

[14] Crawley-Boevey, W.: Geometry of the moment map for representations of quivers. Compositio Math. 126, 257-293 (2001)

[15] Crawley-Boevey, W.: Indecomposable parabolic bundles and the existence of matrices in prescribed conjugacy class closures with product equal to the identity. Publ. Math. Inst. Hautes Études Sci. No. 100, 171-207 (2004)

[16] Crawley-Boevey, W., Shaw, P.: Multiplicative preprojective algebras, middle convolution and the Deligne-Simpson problem. Adv. Math. 201, 180-208 (2006)

[17] Dettweiler, M., Reiter, S.: An algorithm of Katz and its application to the inverse Galois problem. J. Symbolic Comput. 30, 761-798 (2000)

[18] Dettweiler, M., Reiter, S.: Middle convolution of Fuchsian systems and the construction of rigid differential systems. J. Algebra 318, 1-24 (2007)

[19] Dettweiler, M., Reiter, S.: Painlevé equations and the middle convolution. Adv. Geom. 7, 317-330 (2007)

[20] Filipuk, G.: On the middle convolution and birational symmetries of the sixth Painlevé equation. Kumamoto J. Math. 19, 15-23 (2006)

[21] Harnad, J.: Dual isomonodromic deformations and moment maps to loop algebras. Comm. Math. Phys. 166, 337-365 (1994)

[22] Haraoka, Y., Filipuk, G.: Middle convolution and deformation for Fuchsian systems. J. Lond. Math. Soc. (2) 76, 438-450 (2007)

[23] Haraoka, Y., Yokoyama, T.: Construction of rigid local systems and integral representations of their sections. Math. Nachr. 279 255-271 (2006)

[24] Hukuhara, M.: Sur les points singuliers des équations différentielles linéaires, II. J. Fac. Sci. Hokkaido Univ. 5, 123-166 (1937) 
[25] Jimbo, M., Miwa, T., Môri, Y., Sato, M.: Density matrix of an impenetrable Bose gas and the fifth Painlevé transcendent. Phys. D 1, 80-158 (1980)

[26] Katz, N. M.: Rigid local systems. Annals of Mathematics Studies, vol. 139. Princeton University Press, Princeton (1996)

[27] Kawakami, H.: Generalized Okubo systems and the middle convolution. Thesis, The University of Tokyo (2009)

[28] Kostov, V.: The Deligne-Simpson problem—a survey. J. Algebra 281, 83-108 (2004)

[29] King, A. D.: Moduli of representations of finite-dimensional algebras. Quart. J. Math. Oxford Ser. (2) 45, 515-530 (1994)

[30] Le Bruyn, L., Procesi, C.: Semisimple representations of quivers. Trans. Amer. Math. Soc. 317, 585-598 (1990)

[31] Levelt, A. H. M.: Jordan decomposition for a class of singular differential operators. Ark. Mat. 13, 1-27 (1975)

[32] Lusztig, G.: On quiver varieties. Adv. Math. 136, 141-182 (1998)

[33] Mumford, D., Fogarty, J., Kirwan, F.: Geometric invariant theory, 3rd edn. Ergebnisse der Mathematik und ihrer Grenzgebiete (2)34. Springer, Berlin (1994)

[34] Nakajima, H.: Instantons on ALE spaces, quiver varieties, and Kac-Moody algebras. Duke Math. J. 76, 365-416 (1994)

[35] Okamoto, K.: Isomonodromic deformation and Painlevé equations, and the Garnier system. J. Fac. Sci. Univ. Tokyo Sect. IA Math. 33, 575-618 (1986)

[36] Okubo, K.: Connection problems for systems of linear differential equations. In: Urabe, M. (ed.) Japan-United States Seminar on Ordinary Differential and Functional Equations (Kyoto, 1971), Lecture Notes in Math., Vol. 243, pp. 238-248. Springer, Berlin (1971)

[37] Oshima, T.: A quantization of conjugacy classes of matrices. Adv. Math. 196, 124-146 (2005)

[38] Oshima, T.: Classification of Fuchsian systems and their connection problem. arXiv:0811.2916

[39] Turrittin, H. L.: Convergent solutions of ordinary linear homogeneous differential equations in the neighborhood of an irregular singular point. Acta Math. 93, 27-66 (1955)

[40] Völklein, H.: The braid group and linear rigidity. Geom. Dedicata 84, 135-150 (2001)

[41] Weinstein, A.: The local structure of Poisson manifolds. J. Differential Geom. 18, 523557 (1983)

[42] Woodhouse, N. M. J.: Duality for the general isomonodromy problem. J. Geom. Phys. 57, 1147-1170 (2007)

[43] Yokoyama, T.: Construction of systems of differential equations of Okubo normal form with rigid monodromy. Math. Nachr. 279, 327-348 (2006) 